\title{
Working With the Wave Equation in Aeroacoustics- The Pleasures of Generalized Functions
}

\author{
F. Farassat \\ NASA Langley Research Center, Hampton, Virginia \\ Senior Theoretical Aeroacoustician, Fellow AIAA, \\ feri.farassat@nasa.gov \\ Kenneth S. Brentner \\ The Pennsylvania State University, University Park, Pennsylvania \\ Associate Professor, Associate Fellow AIAA, \\ ksbrentner@psu.edu \\ Mark H. Dunn \\ National Institute for Aerospace (NIA), Hampton, Virginia \\ Consultant, Senior Member AIAA, Mhd314@aol.com
}

\section{Abstract}

The theme of this paper is the applications of generalized function (GF) theory to the wave equation in aeroacoustics. We start with a tutorial on GFs with particular emphasis on viewing functions as continuous linear functionals. We next define operations on GFs. The operation of interest to us in this paper is generalized differentiation. We give many applications of generalized differentiation, particularly for the wave equation. We discuss the use of GFs in finding Green's function and some subtleties that only GF theory can clarify without ambiguities. We show how the knowledge of the Green's function of an operator $L$ in a given domain $D$ can allow us to solve a whole range of problems with operator $L$ for domains situated within $D$ by the imbedding method. We will show how we can use the imbedding method to find the Kirchhoff formulas for stationary and moving surfaces with ease and elegance without the use of the four-dimensional Green's theorem, which is commonly done. Other subjects covered are why the derivatives in conservation laws should be viewed as generalized derivatives and what are the consequences of doing this. In particular we show how we can imbed a problem in a larger domain for the identical differential equation for which the Green's function is known. The primary purpose of this paper is to convince the readers that GF theory is absolutely essential in aeroacoustics because of its powerful operational properties. Furthermore, learning the subject and using it can be fun.

\section{Nomenclature}

$C^{\infty}$ : The space of infinitely differentiable functions

$C^{n}$ : The space of continuously differentiable functions up to the order $n$ 
$D$ : The space of infinitely differentiable functions with compact (closed and bounded) support

$D^{\prime}$ : The space of continuous linear functionals on the space $D$, i.e., the space of Schwartz GFs

GD : Generalized derivative

GF : Generalized function

OF : Ordinary function, any function that is locally Lebesgue integrable

$\epsilon$ : Belongs to

$[0,1] \backslash[a, b]:$ Set subtraction, $[0, a] \cup(b, 1]$

\section{1- Introduction}

The linear wave equation and its counterpart in frequency domain, the Helmholtz equation, are the most important partial differential equations of aeroacoustics. For example, both the Lighthill $^{1}$ jet noise equation and the Ffowcs Williams-Hawkings $(\mathrm{FW}-\mathrm{H})$ equation $^{2}$ are linear wave equations. We will primarily be concerned with the wave equation here. Although this equation in one, two, three and higher space dimensions has been analyzed since the eighteenth century ${ }^{3}$, there are still many issues and subtleties associated with finding the solution, particularly in aeroacoustics, that are in need of further attention. It is true to say, and this fact is well-known to mathematicians, that the appropriate mathematical tool for solving PDEs is the theory of distributions or generalized functions (GFs) ${ }^{3-12}$. The derivation and finding the solution of the FW-H equation depend heavily on the concept of GFs which requires much mathematical maturity from the users. Furthermore, even when we use the Lighthill equation, we may be dealing with flowfields that have discontinuities within them, such as shocks, which can be sources of sound. The natural tool to work with these discontinuities is GF theory. The principal aim of this paper is to give a working knowledge of the multidimensional GF theory to acousticians, particularly when using the linear wave equation.

There are many books and papers on the mathematics of the wave equation and the related problems that are used by acousticians. Unfortunately, almost all of the commonly referenced books and papers were written before, or without the use of, the theory of distributions (generalized functions) of Laurent Schwartz ${ }^{4}$. These books and papers use an intuitive approach to Dirac delta function and its algebra that is not rigorous and is prone to giving erroneous results. Furthermore, much of the true power of GF theory is not apparent from the intuitive approach. The available books on mathematics that discuss generalized functions (GFs) rigorously ${ }^{4-6}$ are also beyond the reach of most acousticians because of the level of abstraction involved to comprehend the theory. We believe that most of the abstractions are not necessary for application of the GFs. The situation is much like the use of the real numbers by the man in the street in everyday business without ever knowing about Cauchy convergent sequences because he only needs to know the useful properties of the real numbers. For mathematically minded persons the abstractions in the development of the theory can be learned most easily after a working knowledge of GFs is attained.

We will start by giving the definition of GFs as continuous linear functionals on a given space of functions. We will do this in the simplest possible way. We will then discuss how the definition of many useful operations on ordinary functions can be extended to GFs. Of particular interest to us here is the definition of generalized derivative and its many useful applications. These include the Green's functions of ODEs and PDEs, finite part of divergent integrals, the Kirchhoff formula for moving surfaces and getting the jump condition from conservation laws. We will discuss some questions associated with the derivation of the $\mathrm{FW}-\mathrm{H}$ equation. In particular, we answer the question of whether any function 
other than the null function can be assumed inside the moving surface in the derivation of the FW-H equation. We will see that in every aspect of working with the linear wave equation, the natural mathematical tool is the GF theory.

We wish to convey to the readers our enthusiasm of working with GFs as the most essential, useful and powerful tool for application in acoustics. We have, therefore, chosen a subtitle for this paper with an approving nod to the title of the delightful and scholarly book of Thomas Körner ${ }^{13}$.

\section{2- Generalized Functions (GFs)}

In this section we will first start by giving the definition of GFs. We then present the most common operations on GFs. We will pay a special attention to generalized differentiation which is the fundamental concept for obtaining the Green's function, finding the finite part of divergent integrals and working with jump conditions from conservation laws. We will then give many applications of GFs relevant to acoustics. We will say little about generalized Fourier transformation because that subject by itself requires a full paper.

In learning GF theory, you should know that there are several different approaches to the definition of GFs. The method we present here is the functional approach of Schwartz ${ }^{4-11}$. The sequential approach of Temple and Lighthill ${ }^{14}$, Jones ${ }^{12}$ and Mikusinski ${ }^{15}$. The completeness theorem of GFs relates the two approaches ${ }^{7}$. The approach by Bremermann defines GFs as the boundary value of analytic functions ${ }^{16}$. This subject is valuable in studying generalized Fourier analysis and, in its full generality, requires the knowledge of the highly specialized topic of functions of several complex variables. Most of Bremermann's book ${ }^{16}$ does not require this topic and for learning about one dimensional GFs, it is easily accessible to readers with a good knowledge of analytic functions of a single complex variable. Eventually, when you work on advanced Fourier transform theory, you will have to learn Bremermann's approach because it sheds light on the derivation of many of the results obtained by physicists and applied mathematicians using ad hoc and mostly intuitive reasoning. The subject can be shown to be also closely related to the functional approach we use here but we will not say any more about this connection ${ }^{7}$.

The Polish mathematician Jan Mikusinski introduced one more method of defining GFs known as the operational approach which is based on the concepts from abstract algebra ${ }^{17,18}$. Mikusinski's publications are beautifully written and are easy to read and comprehend. Apart from explaining rigorously the Heaviside operational calculus, this approach can also be used to solve an entirely different set of problems than the first three approaches, such as finding the exact solutions to finite difference equations and recursion relations. The relationship between the Mikusinski's operational approach and the functional approach cannot be established easily and we are not aware of any publications on the subject. We encourage the readers to study this approach because of its usefulness and power in solving some important problems of physics and engineering ${ }^{17-20}$. We will say no more on this approach here.

And finally, the readers should be aware of the fact that in recent years GF theory has been extended to the so-called nonlinear GF (NLGF) theory where some of the limitations of Schwartz distribution (GF) theory are removed ${ }^{21-23}$. For example, it can be shown that one cannot extend the operation of multiplication to all of Schwartz GFs. This shortcoming of the Schwartz theory does cause some difficulties in applications that Colombeau ${ }^{21}$, Rosinger $^{22}$ and Oberguggenberger ${ }^{23}$ as well as other mathematicians have tried to resolve. Currently, the NLGF theory is very abstract and difficult to master. The subject is under intense development but is badly in need of simplification. However, it appears that the subject of 
nonstandard analysis (NSA) ${ }^{24}$ will eventually be able to clarify and simplify NLGF theory considerably and bring the theory within the reach of physicists and engineers ${ }^{25}$. We will not cover this subject here. The readers should know that to understand NLGF theory and NSA, requiring one to have a knowledge of several esoteric areas of mathematics including topology, abstract algebra and mathematical logic. Fortunately, there are many expository books and articles on these subjects on the internet, particularly on NSA. We will not cover this theory here.

For those interested in the history of GF theory, we recommend the books by Lützen ${ }^{26}$ and Dieudonné $^{27}$ as well as the article by Synowiec ${ }^{28}$. In the opinion of Lützen, the Russian mathematician Sobolev invented the concept of GFs (more exactly, generalized derivatives) and Schwartz developed the theory of GFs without being aware of Sobolev's earlier contributions ${ }^{4}$. As expected, many other mathematicians contributed to the field. Without a doubt, the members of the Moscow School of Mathematics under I. M. Gelfand have contributed substantially to the development and applications of GF theory making the subject accessible to physicists and engineers. The well-known book on GFs by Gelfand and Shilov $^{7}$ is the first of a six volume opus by this School published in the West in the sixties of the last century. This monumental work, which is still read today, has popularized the GF theory enormously among physicists and mathematicians by its breadth and depth as well as by the clarity of the exposition. For learning about more recent advances we suggest the books by Hörmander ${ }^{5}$ and Taylor ${ }^{6}$ which are both written for mathematicians.

Most of this section is based on the first author's expository NASA publication ${ }^{29}$. In addition to this publication, we recommend that you consult the books by Strichartz ${ }^{10}$, Stakgold $^{8}$, Gelfand and Shilov ${ }^{7}$, and Kanwal ${ }^{11}$. For beginners, we particularly recommend the excellent book by Strichartz for deeper understanding of many of the concepts discussed here. This book emphasizes GFs in one dimension which is essential in some applications, e.g., time series analysis. However, there are many problems of physics and engineering for which multidimensional GF theory is very important ${ }^{7-9,11,12,29}$. This is the subject that we concentrate in the present paper. The emphasis will be on using the theory to solve problems rather than stating theorems and giving proofs.

\section{1- Definition of Generalized Functions (GFs)}

What we call a function, say $f(x)$, in calculus can be thought of as a table of ordered pairs $(x, f(x))$. We can visualize this table as a graph of $x$ versus $y=f(x)$. If we want to generalize the concept of a function, we must change the definition of a function in such a way that our old functions are still considered functions and we have many new useful mathematical objects that are functions by the new definition. Once we introduce a new definition of a function, we must make sure that we can extend all the useful operational properties of the old functions, e.g., addition of two functions, to all the new mathematical objects which we now collectively (the old functions and the new objects) call generalized functions $(G F s)$. For now, we refer to all the functions that we learned about in calculus as ordinary functions (OFs). We will elaborate further on this below.

Let us start by considering an ordinary function $g(x)$ which is periodic over the interval $[0,2 \pi]$. We know that the complex Fourier components of this function are given by the relation

$$
G_{n}=\frac{1}{2 \pi} \int_{0}^{2 \pi} g(x) e^{-i n x} d x \quad(n=-\infty \text { to } \infty)
$$


We know that $g(x)=\sum_{n=-\infty}^{\infty} G_{n} e^{i n x}$, that is, the periodic function $g(x)$ can be constructed from the knowledge of the table with infinite entry $\left\{G_{n}, n=-\infty\right.$ to $\left.\infty\right\}$. From another point of view, the set of complex numbers $\left\{G_{n}\right\}$ can be considered as the mapping of the space of functions $\left\{e^{i n x}, n=-\infty\right.$ to $\left.\infty\right\}$ into complex numbers by the rule given in eq. (1). Such a mapping from a given space of functions into scalars (real or complex numbers) is called a functional. Remember that a functional is always a rule that describes the mapping from a given space of functions into scalars. The numbers $\left\{G_{n}\right\}$ are the functional values of this functional. Note that the function $g(x)$ enters the definition of the functional. As a matter of fact, we should use the symbol $G_{n}[g]$ to remind us that the number $G_{n}$ depends on the function $g(x)$. So far we have shown that the periodic function $g(x)$ can be described (identified, defined) by the table of its functional values:

$$
\left\{G_{n}[g]=\frac{1}{2 \pi} \int_{0}^{2 \pi} g(x) e^{-i n x} d x, n=-\infty \text { to } \infty\right\} \text { on the space }\left\{e^{i n x}, n=-\infty \text { to } \infty\right\}
$$

We have assumed here that the function $g(x)$ has a Fourier series (e.g., satisfies the Dirichlet conditions, etc.). Now we have the germ of an idea to extend the definition of a function.

Let us take a space of functions of one variable $D$ with a large number of well-defined functions in the space. We will say more about the properties of the functions in this space soon. Let us select an ordinary function $f(x)$ and for any function $\phi(x)$ in the space $D$, define the functional (i.e., the rule):

$$
F[\phi]=\int_{-\infty}^{\infty} f(x) \phi(x) d x \quad(f \text { is fixed in this rule and } \phi \text { varies all over } D)
$$

Note carefully how the function $f(x)$ enters the definition of the functional. To describe $f$, this function is kept fixed in the rule given by eq. (3) and different functions $\phi$ are taken (conceptually, of course, not literally) from the space $D$ to form the table $\{F[\phi], \phi \in D\}$. Therefore, we must have so many functions in space $D$ that any two different ordinary functions generate different tables of functional values at least for some entries. The space $D$ is known as the test function space. The question is how to select the space $D$ in such a way that the set of the functional values $\{F[\phi], \phi \varepsilon D\}$ represents (i.e., describes or has as much information content as) any ordinary function $f(x)$. Before we do this, we must now elaborate on what we mean by ordinary functions.

By ordinary functions (OFs), we mean the locally Lebesgue integrable functions. This means that they are functions that are Lebesgue integrable ${ }^{7-11,19}$ over any finite interval. These functions include any conceivable functions, no matter how wild, that are needed in applications. If you do not know Lebesgue integration, you can think of Riemann integration that you learned in calculus courses. Lebesgue integration is a generalization of the Riemann integration that can be used for the integration of most functions that do not have Riemann integral.

One can show that there is an uncountable number of OFs. Therefore, the space of test functions must contain an uncountable number of functions so that any two different OFs can be distinguished from each other when viewed as tables of functional values. This means that our tables of functional values used to represent OFs are also uncountable. Furthermore, since OFs are as wild as they can be, we require that the functions in space $D$ to be as well-behaved as they can be so that the integral in eq. (3) is always defined. This leads us to require that the functions in $D$ to be infinitely differentiable (denoted $C^{\infty}$ functions). Also to ensure the integral in eq. (3) does not become infinite, we assume that all functions in space $D$ are identically zero outside of a finite interval. This interval can be different for differ- 
ent test functions. A function that is identically zero outside some finite interval is said to have bounded support. Thus, we have come to the following conclusion regarding the space of test functions $D$ that we need to use to identify OFs by their tables of functional values:

The test function space $D$ that one needs to use in defining OFs by their tables of functional
values is the space of all $C^{\infty}$ functions with compact (closed and bounded) support.

Let us say something about this space next. The best known, and in many ways the fundamental, example of a function in $D$ is the following bell-shaped function with the peak value at the origin. It is defined for any real number $a>0$ :

$\phi(x ; a)= \begin{cases}e^{x^{2} /\left(x^{2}-a^{2}\right)} & |x|<a \\ 0 & |x| \geqslant a\end{cases}$

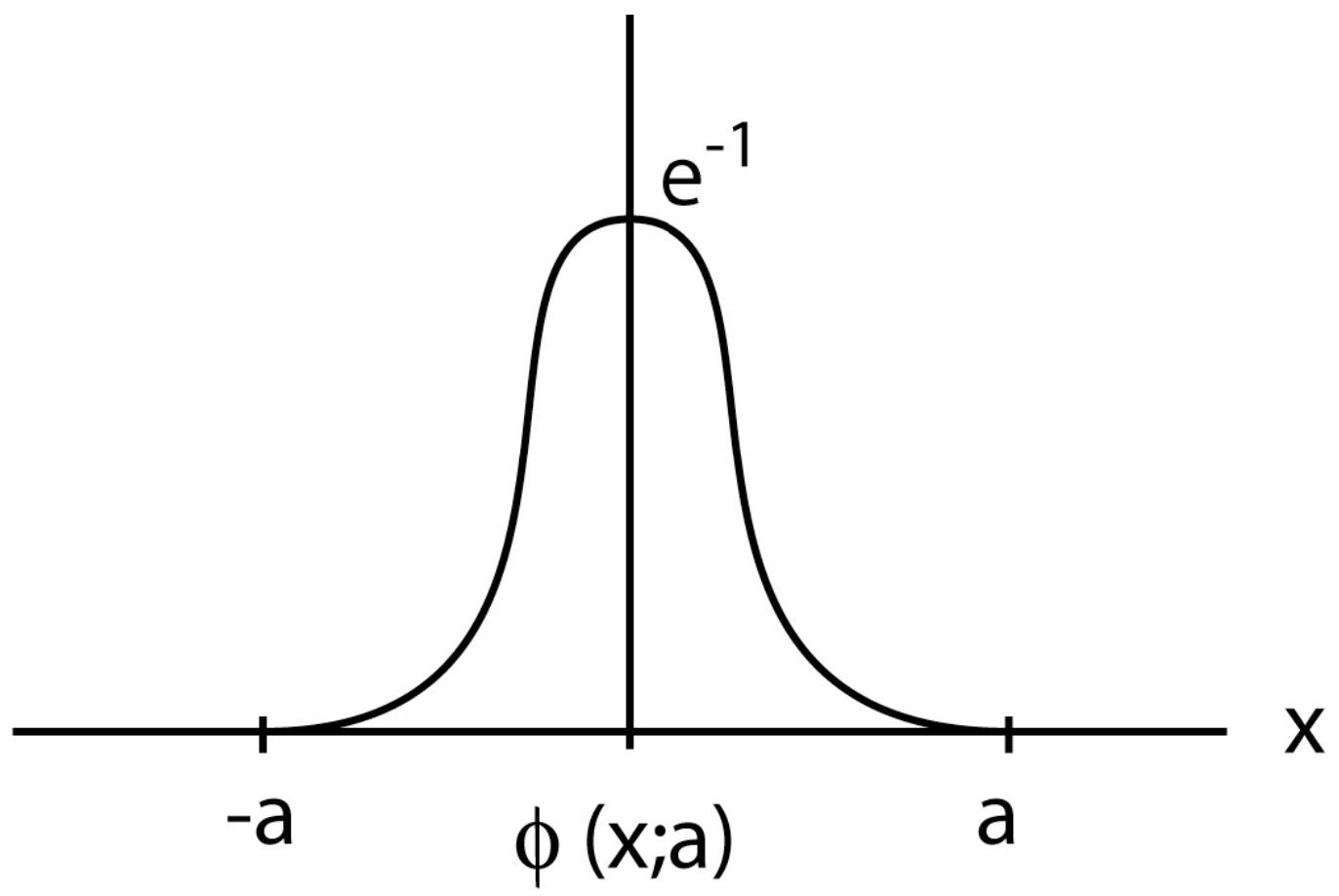

Figure 1- The Graph of the function $\phi(x ; a)$

It is easy to see that the support of this function is $[-a, a]$ and it is infinitely differentiable on the open interval $(-a, a)$. Figure 1 shows the graph of this function. The proof of infinite differentiability at points $x=-a$ and $x=a$ is much harder and is an exercise in calculus albeit requiring much technical skill. You should know that at these two points all the derivatives of this function are zero. Are there any other types of functions in space $D$ besides $\phi(x ; a)$ ? The answer is affirmative and we will turn to this point next.

One proves rigorously in real analysis that there is an uncountably infinite number of continuous functions. For any continuous function $g(x)$ defined on the bounded interval $\mathrm{I}=[b, c]$, the convolution of $g(x)$ and $\phi(x ; a)$ on $I$ gives a $C^{\infty}$ function with the bounded support $[b-a, c+a]^{19}$. Therefore, there is an uncountable number of functions in the test function space $D$ as we are required to have. Another fact to remember about this space is that the functions in space $D$ are not analytic even though they are $C^{\infty}$ functions! 
At this stage, we have a new way of looking at OFs but our original intention was to extend the definition of a function so that we have new objects that are not OFs but are considered functions. To do this we study two important properties of the functionals described by eq. (3). The first one is very easy to see:

$1-$ The functional $F[\phi]$ is linear.

This simply means that for $\alpha$ and $\beta$ scalars and $\phi$ and $\psi$ functions in $D$, we have

$$
F[\alpha \phi+\beta \psi]=\alpha F[\phi]+\beta F[\psi]
$$

To show this, replace $\phi(x)$ on the right of eq. (3) with $\alpha \phi+\beta \psi$ and use the linearity property of integrals followed by functional interpretation of the result to get the right side of eq. (4). We remind the readers that the $\mathrm{OF} f(x)$ used in the definition of the functional of eq. (3) is fixed here and the functional $F[\phi]$ is, in point of fact, representing $f(x)$.

The second property is much harder to explain and comprehend. It is:

2 - The functional $F[\phi]$ is continuous.

The first thing you must know is that this property, whatever it means, gives GFs a lot of useful properties that we need in applications. Second, to the beginners the definition of continuity of a functional appears too complicated and perhaps a futile attempt in abstraction. We admit that the definition of the continuity of a functional is complicated but its definition cannot be stated in simpler form than we give here. The original definition by Schwartz was much more complicated and based on locally convex topological spaces ${ }^{4}$. Just remember that you will appreciate the full usefulness of this property when you learn some more about GFs and their applications. See, for instance, Example 3 below.

We first distinguish some sequences of functions $\left\{\phi_{n}\right\}$ in $D$, which we say they go to zero in $D$, and write it as $\phi_{n} \stackrel{D}{\longrightarrow} 0$. For any such sequence, we require that it satisfies two conditions:

i- There is a bounded interval $J$ such that the support of $\phi_{n} \subset J$ for all $n$, and

ii- $\phi_{n}$ and all its derivatives go to zero uniformly in $J$.

We make two useful comments here concerning these conditions. First, in general, different sequences going to zero in $D$ have different bounded interval $J$ associated with them. In other words, we require only that for a given sequence going to zero in $D$ such a bounded interval to exist. Second, condition ii simply means that if $m$ is the order of the derivative, $m=0,1,2, \ldots .$, and given $\varepsilon>0$, we can find $N(m)$ such that for any $x$ in the interval $J$, we have $\left|\phi_{n}^{(m)}(x)\right|<\varepsilon$ for all $n>N(m)$. Note that the word "uniformly" means that the number $N(m)$ is independent of $x$ but it can depend on the order of differentiation $m$. Here we have used the notation $\phi_{n}^{(m)}(x)$ for the $m$ th derivative of $\phi_{n}(x)$.

Example 1- We can show easily that the following sequence goes to zero in $D$ :

$\phi_{n}(x)=\frac{1}{n} \phi(x ; a) \quad($ See eq. (4) for the definition of $\phi(x ; a))$

Example 2- The following sequence of functions does not go to zero in $D$ :

$\phi_{n}(x)=\frac{1}{n} \phi\left(\frac{x}{n} ; a\right) \quad$ (See eq. (4) for the definition of $\left.\phi(x ; a)\right)$ 
The reason is that for a given function $\phi_{n}$, the support is the interval $[-n a, n a]$ and, therefore, the support cannot remain bounded for all $n$. Condition ii is, thus, violated.

Now we can give the definition of continuity of a functional:

$A$ functional $F[\phi]$ is continuous if for any $\phi_{n} \stackrel{D}{\longrightarrow} 0$, we have $F\left[\phi_{n}\right] \longrightarrow 0$.

Note that $\left\{F\left[\phi_{n}\right]\right\}$ is simply a sequence of real or complex numbers. By using the properties of (Lebesgue) integrals, we can show the following very important result:

Any OF $f(x)$ generates a continuous linear functional through

the relation : $F[\phi]=\int_{-\infty}^{\infty} f(x) \phi(x) d x \quad(\phi$ is in the test function space $D)$

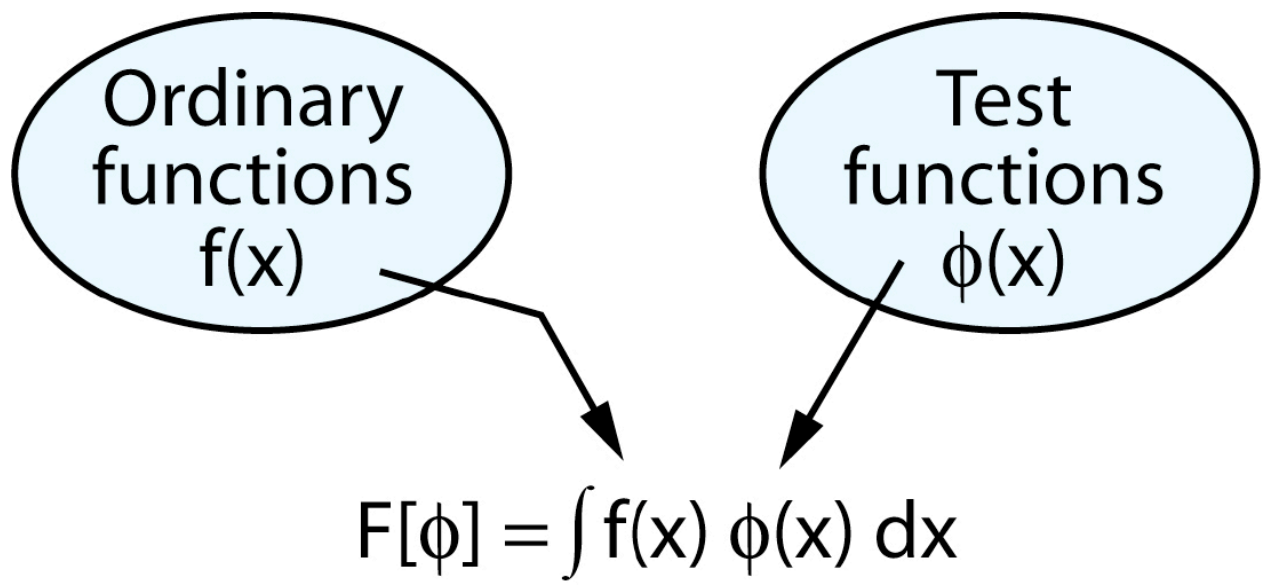

Figure 2- The functional rule used to define an OF $f(x)$ by a table of its functional values over the space $D$

Figure 2 shows schematically how the table of functional values is constructed for an OF. So we can use the above functional (or rule) to construct the sequence or the table of numbers $\{F[\phi], \phi \in D\}$ to identify (or describe, distinguish) the OF $f(x)$. Are there any more continuous linear functionals described by other rules? The answer is affirmative and, in fact, there are many of them. Let us consider the following example:

Example 3- Define the functional $\delta[\phi]=\phi(0), \phi \in D$. We can easily show that this functional is linear. We now show that it is also continuous. Take any sequence that goes to zero in $D$, say $\left\{\phi_{n}\right\}$. Since $\phi_{n} \stackrel{D}{\longrightarrow} 0$, then in particular $\phi_{n} \longrightarrow 0$ uniformly. The uniformity implies that it does not matter where $x$ is. Therefore, $\phi_{n}(0)=\delta\left[\phi_{n}\right] \longrightarrow 0$. Can you see how we use condition ii of the definition of continuity of a functional here? Because of the importance of this functional in GF theory, we will give it the name of the Dirac functional. This is obviously related to the Dirac delta function. We will establish the connection below (see eq. (9)).

Using elementary Lebesgue integration theory, we can show that there is no OF $\delta(x)$ that can generate the continuous linear functional $\delta[\phi]$ by using the rule given in eq. (3). Therefore not all the continuous linear functionals are generated by OFs based on eq. (3)!

Now if we decide to define (or describe, identify) any OF conceptually by the table of its functional values using the rule of eq. (3), we may just as well say that the table generated by any continuous 
linear functional also defines a function. We now have new objects (tables) that cannot be identified with OFs, e.g., the functional in Example 3. We call the entire collection of objects (tables of functional values) generalized functions (GFs). We have achieved our goal of generalizing the definition of OFs. We, thus, state the following:

Generalized functions are defined by the tables of functional values of continuous linear functionals.

Since we are now going to think of functions in the new way by their functional values, we will use a slight abuse of language and say:

Generalized functions are continuous linear functionals.

The particular space of GFs that we have constructed using the test function space $D$ is known as the (Schwartz) GF space $D^{\prime}$. We know that by construction, all OFs are also GFs. We refer to OFs as regular generalized functions. The new objects (functions) that cannot be identified as OFs are called singular generalized functions (Think of the GF in Example 3 now.). Figure 3 shows how one should think of the space of GFs. In applications, several other test function spaces are used but the idea behind the construction of the associated GF spaces is exactly the same as we have presented above. At this stage, it is important to know that there are many singular GFs that are absolutely essential in applications $^{7}$. As a matter of fact, the introduction of the singular GFs into mathematics and the development of a rigorous operational algebra to work with them have provided a very powerful analytical tool to physicists and engineers.

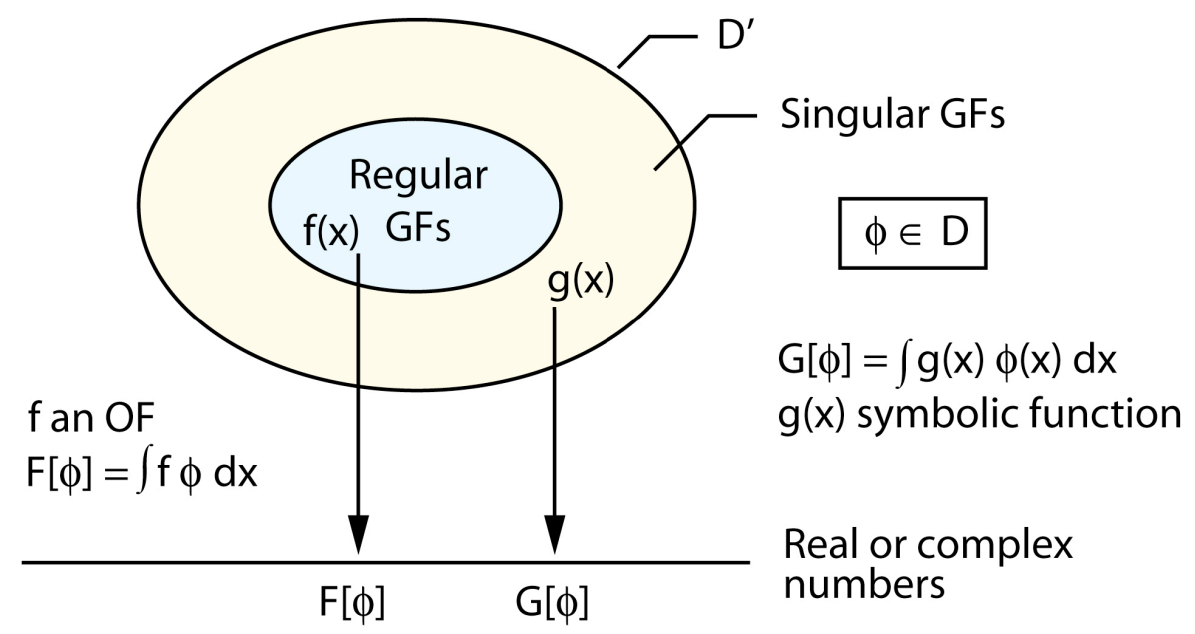

Figure 3- The space $D^{\prime}$ of GFs consisting of regular GFs (OFs) and singular GF that are new

The readers should be aware that the definition of GFs given above, like the construction of real numbers by Cauchy convergent sequences in analysis, is of enormous conceptual value. We will have to use this definition to obtain the properties of GFs and to extend the useful operations on OFs to all GFs. But once we have achieved these goals, we want to get away from dealing with functionals as functions and work with GFs with the same notations and ease as OFs. Like the man in the street who never needs to know about convergent sequences to work with numbers, we want to use GFs without being burdened by the conceptual framework of the construction of GFs. We can actually do this but first let us look at an example of a GF.

Example 4- Here is a continuous linear functional defining a GF: 


$$
G[\phi]=\int_{-\infty}^{\infty} e^{2 x} \sin 3 x \phi(x) d x-5 \phi(0) \quad \phi \in D
$$

You should have little problem to prove this fact. Note that, in practice, we always give the rule (the definition of the functional) only and we do not attempt to construct the table of the functional values because it is technically impossible. But we can use the rule to make part of the table if we want to!

We will now dispose of the functional notation by the introduction of symbolic functions. This is a brilliant idea. It will allow us to work with singular generalized functions just like the OFs. We start with the Dirac functional of Example 3 which is a singular GF. We introduce a symbolic function $\delta(x)$ which is supposed to hold the information (or the memory) about the Dirac functional when we are doing algebraic manipulations until it appears as an integrand of an integral in a product with a test function. We then use the following relation agreed upon by convention:

$$
\int_{-\infty}^{\infty} \phi(x) \delta(x) d x \equiv \delta[\phi]=\phi(0) \quad \phi \in D
$$

It is very important to recognize that 'the integral' on the left of this equation is not a Riemann or a Lebesgue integral because $\delta(x)$ is not an OF. You should think of the whole integral symbol like a Chinese character standing for $\delta[\phi]=\phi(0)$ which is now completely meaningful. The symbolic function $\delta(x)$, known universally as the Dirac delta function, can be used in algebraic manipulations like an OF until it appears under an integral sign like that on the left of eq. (9). You should, however, learn some more about the properties of singular GFs before you can comfortably or boldly manipulate them algebraically. We will give you what you need to know later but first let us look again at Example 4 using symbolic function notation.

Example 5- The GF in Example 4 can be written symbolically as $g(x)=e^{2 x} \sin 3 x-5 \delta(x)$. This GF is the sum of a regular and a singular GF.

\section{2 - Multidimensional GFs}

The above definition of GFs can easily extended to multidimensions. The test function space $D$ is now n-dimensional and consists of all infinitely differentiable functions with bounded support. An example of such a function can be constructed from the function in eq. (4). Let $\boldsymbol{x}=\left(x_{1}, x_{2}, \ldots ., x_{n}\right)$, and $|\boldsymbol{x}|=\left(x_{1}^{2}+x_{2}^{2}+\ldots+x_{n}^{2}\right)^{1 / 2}$. Then the we can show that for $a>0$, the following function is in space $D$ :

$\phi(x ; a)= \begin{cases}e^{|\boldsymbol{x}|^{2} /\left(|\boldsymbol{x}|^{2}-a^{2}\right)} & |\boldsymbol{x}|<a \\ 0 & |\boldsymbol{x}| \geqslant a\end{cases}$

From this function and any continuous function, we can generate another test function by convolution over a finite region of space. Therefore, the space of test functions $D$ has an uncountably many functions in it. We then define the multidimensional (Schwartz) GF space $D^{\prime}$ as the space of continuous linear functionals on $D$. This definition is appropriate for discovering the operational properties of GFs that give us a powerful tool for applications. In practice, however, we will work with symbolic functions when we deal with singular generalized functions. Therefore, we will be always working with generalized functions as if we are working with OFs. Intuitively, this is of great help to people interested in 
applications. The situation is similar to the use of identical notations for real and complex numbers as much as possible when we are solving a problem in complex plane.

From our point of view, the multidimensional generalized functions are much more important than GFs of one variable in studying partial differential equations and in particular wave propagation problems. It is important to recognize that to study the multidimensional GFs, one needs to have a working knowledge of differential geometry of curves and surfaces ${ }^{30}$.

As in the case of one variable, the most important singular GFs in multidimensions are the Dirac delta function and its derivatives. Of interest to us is the delta function with the support on a surface which can be in motion. This function has no one dimensional analogue. We start with a simple example and then discus the Dirac delta function with the support on a surface.

Example 6- The Dirac delta function $\delta(\boldsymbol{x})$ with support at the origin has the following property

$$
\int_{V} \phi(\boldsymbol{x}) \delta(\boldsymbol{x}) d \boldsymbol{x}=\phi(0) \quad \phi \in D
$$

where $V$ is an arbitrary volume which includes the origin.

Example 7- Referring to Figure 4, let $S: f(\boldsymbol{x})=0$ be a smooth surface defined in such a way that the unit outward normal $\boldsymbol{n}=\nabla f$. This can always be done because if $\boldsymbol{n} \neq \nabla f$, then define this surface by the new implicit function $f(\boldsymbol{x}) /|\nabla f|=0$ which has the desired property.

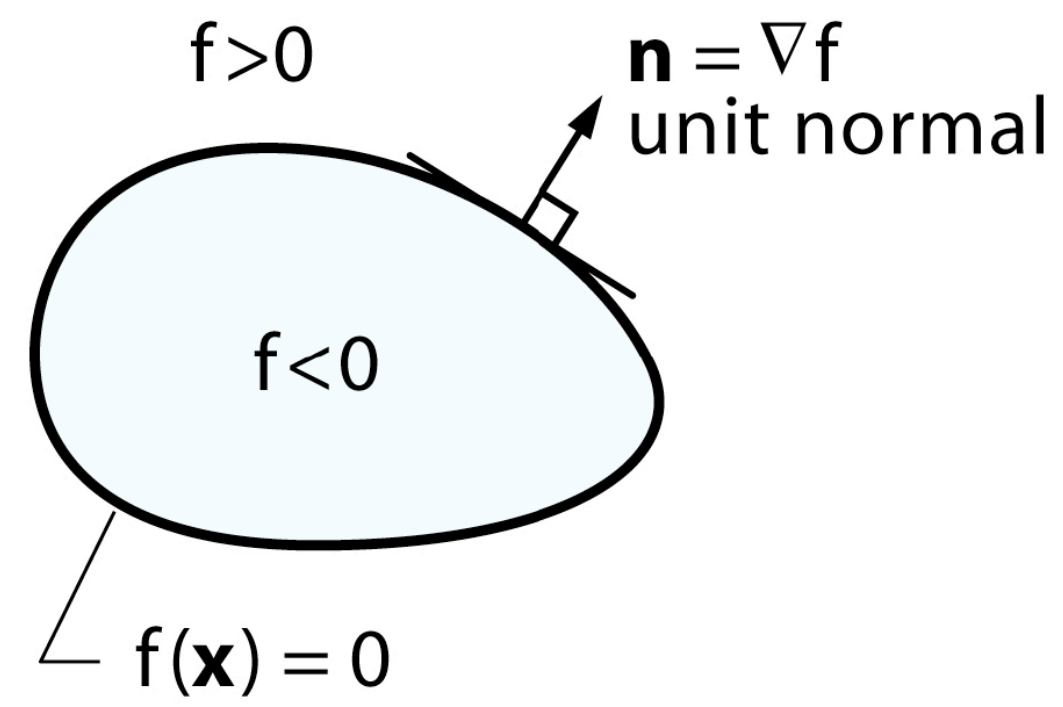

Figure 4- The description of the surface $\mathbf{S}$ defined implicitly by $f(\boldsymbol{x})=0$ with unit outward normal $n=\nabla f$.

We want to interpret the important GF $\delta(f)$ often appearing in applications. We define a Gaussian curvilinear coordinates $\left(u^{1}, u^{2}\right)$ over the surface $S$ and define the third coordinate $u^{3}=f$ along the local normal to $S$ to all points in the space in the vicinity of $S$. Note that we have

$$
d \boldsymbol{x}=\sqrt{g_{(2)}(\boldsymbol{u})} d \boldsymbol{u} \quad \boldsymbol{u}=\left(u^{1}, u^{2}, u^{3}\right)
$$

where $g_{(2)}(\boldsymbol{u})$ is the determinant of the first fundamental form of the surface $u^{3}=f(\boldsymbol{x})=$ constant $^{29-31}$. We thus have the following very useful result 


$$
\begin{aligned}
& \int \delta(f) \phi(\boldsymbol{x}) d \boldsymbol{x}=\int \delta\left(u^{3}\right) \phi(\boldsymbol{x}(\boldsymbol{u})) \sqrt{g_{(2)}(\boldsymbol{u})} d u^{1} d u^{2} d u^{3}= \\
& \int \phi\left(\boldsymbol{x}\left(u^{1}, u^{2}, 0\right)\right) \sqrt{g_{(2)}\left(u^{1}, u^{2}, 0\right)} d u^{1} d u^{2}=\int_{f=0} \phi(\boldsymbol{x}) d S
\end{aligned}
$$

You can see the importance of differential geometry here. Note that we have used the definition of the Dirac delta function in one variable when we integrate with respect to variable $u^{3}$ after the second equality sign. For a more geometric derivation of this result see the papers by Farassat ${ }^{29,31}$.

It is important to remember that one cannot define a GF at a single point but over a finite interval. This does not cause any difficulties in applications. We can talk about equality of two GFs over an interval. We say that two GFs $F[\phi]$ and $G[\phi]$ are equal over an interval $I$ if for all $\phi \epsilon D$ with support fully within $I$, we have $F[\phi]=G[\phi]$. Remember, a functional is a rule that gives us a number for any function in $D$. Therefore, we are talking about equality of two numbers obtained from two rules. From this definition we can say that $\delta(x)=0$ (the zero function and not the real number zero) over the interval $(-\infty,-\varepsilon] \cup[\varepsilon, \infty)$ for any $\varepsilon>0$.

To learn more about the multidimensional GFs we recommend the books by Gelfand and Shilov ${ }^{7}$, Stakgold $^{8}$, Vladimirov ${ }^{9}$, Kanwal ${ }^{11}$, Jones ${ }^{12}$, Fenyo and Frey ${ }^{19}$, and the two NASA papers by Farassat $^{29,31}$. For more advanced treatment as well some very important recent advances, see the books by Hörmander ${ }^{5}$ and Taylor ${ }^{6}$.

\section{3- Operations on GFs}

Now that we have extended the definition of ODs to GFs, we must extend the definitions of operations on OFs to GFs. The general approach is as follows. Write the definition of the operation in the language of linear functionals and then use it for all GFs if it makes any sense (see the definition of generalized Fourier Transform below where we have to change the test function space). In some cases the extension of the definition of an operation gives us the opportunity to solve new problems or solve old problems in much simpler ways. We single out generalized differentiation here as an example of an operation of considerable importance in applied mathematics.

In the following we assume that all test functions are in space $D$.

\subsection{1- Simple Operations}

1- Addition of two GFs- If $f(x)$ and $g(x)$ are two OFs described by functionals $F[\phi]$ and $G[\phi], \phi \epsilon D$ then using the linear property of integrals, we can write

$$
\int(f+g) \phi d x=\int f \phi d x+\int g \phi d x=F[\phi]+G[\phi]
$$

Basically, we know the left side of the first equality is the functional representation of the sum of the OFs $f(x)$ and $g(x)$ and is given by the right side of the last equality sign. The above result now makes sense for all GFs, i.e., we define

$$
(F+G)[\phi]=F[\phi]+G[\phi]
$$


Note that $(F+G)$ is a single functional which stands for the sum of the two GFs.

Written in symbolic function notation, there is no difference between the notation for OFs and GFs for the sum of two functions. For example, for the sum of an OF $f(x)$ and the Dirac delta function we can simply write $f(x)+\delta(x)$.

2- Multiplication of two GFs- This operation cannot be extended to all of Schwartz GFs. In fact it is the aim of the new nonlinear GF theory to be able to define multiplication for all GFs ${ }^{22,23}$. Any two OFs can be multiplied. We can define multiplication of any GF with any $C^{\infty}$ function. Again let $f(x)$ be any $\mathrm{OF}$ and let $a(x) \in C^{\infty}$, then

$$
\int a(x) f(x) \phi(x) d x=\int f(x)(a(x) \phi(x)) d x=F[a \phi]
$$

We know that the left side of the first equality sign is the functional representation of the OF $a(x) f(x)$. Since the function $a \phi \in D$, the expression on the right of the third equality sign is a functional on the test function space $D$. The rule of multiplication of a generalized function $F[\phi]$ and a $C^{\infty}$ function $a(x)$ is given by the rule

$$
a F[\phi]=F[a \phi]
$$

Note that in this equation, the symbol $a F$ stands for a single functional, i.e., a rule described by the right side of eq. (17). Again in symbolic notation we simply write $a(x) f(x)$ but now we have a rule to interpret such products when $f(x)$ is a singular GF. We give an example here.

Example 8- What is $a(x) \delta(x)$ where $a \epsilon C^{\infty}$ ? Let $\phi \epsilon D$, then

$$
a \delta[\phi]=\int a(x) \delta(x) d x=\delta[a \phi]=a(0) \phi(0)=\int a(0) \delta(x) d x
$$

We can write this result in symbolic function notation as

$$
a(x) \delta(x)=a(0) \delta(x)
$$

This useful result has been known for many years to physicists.

3- Translation of a GF- Let $f(x)$ be an OF, and let us define $E_{h} f(x)=f(x-h)$, i.e., translating the function to the right for $h>0$. In functional notation, we have

$$
\int E_{h} f(x) \phi(x) d x=\int f(x-h) \phi(x) d x=\int f(y) \phi(y+h) d y=F\left[E_{-h} \phi\right]
$$

Again the left side of the first equality sign is the functional generated by $E_{h} f(x)$ for which we use the symbol $E_{h} F[\phi]$. Since for $\phi \in D$, we have $E_{-h} \phi \in D$. Therefore, we use the following result for the definition of the translation of any GF:

$$
E_{h} F[\phi]=F\left[E_{-h} \phi\right]
$$

Once again we have obtained a nontrivial result. In symbolic function notation we use the same notation for GFs as for OFs but occasionally we have to resort to eq. (21) to interpret the exact meaning of the translated GF such as in the following example. 
Example 9- What is $\delta(x-a)$ where $a$ is a real constant? The functional representation of this GF is obviously $E_{a} \delta[\phi]$. Using the above rule, we have

$$
\int \delta(x-a) \phi(x) d x=E_{a} \delta[\phi]=\delta\left[E_{-a} \phi\right]=\delta[\phi(x+a)]=\phi(a)
$$

Again this result has been known by physicists for a long time.

We can extend the definition of other simple operations on OFs to GFs such as the expansion and contraction of the scale of the independent variable. For example, one can rigorously show that for $a \neq 0$ we have the following useful result

$$
\delta(a x)=\frac{1}{|a|} \delta(x)
$$

\subsection{2- More Advanced Operations}

We will extend the definition of two operations on OFs to GFs here. These are Fourier transformation and differentiation operations.

1- Fourier Transform of GFs- We work in one dimension here. Let $f(x)$ be a function defined on $\mathbb{R}$ which has Fourier transform define by the relation

$$
\hat{f}(y)=\int_{-\infty}^{\infty} f(x) e^{2 \pi i x y} d x
$$

If the functional $F[\phi]$ is identified with $f(x)$, then its Fourier transform must be identified with $\hat{f}(y)$ as follows

$$
\hat{F}[\phi]=\int_{-\infty}^{\infty} \hat{f}(y) \phi(y) d y=\int_{-\infty}^{\infty} f(y) \hat{\phi}(y) d y=F[\hat{\phi}]
$$

where $\hat{\phi}$ is the Fourier transform of $\phi$. We have used Parseval's theorem after the second equality sign. Now the functional $F[\hat{\phi}]$ is only defined if $\hat{\phi} \in D$ whenever $\phi \in D$. We can show that, in general, this is not so and $\hat{\phi}$ can lie outside the space $D$. We need to define a test function space $S$ on $\mathbb{R}$ consisting of $C^{\infty}$ functions which go to zero at infinity faster than $\left|x^{-n}\right|$ for any $n$. This can be done and in fact we can show that $D \subset S$. The space of tempered GFs $S^{\prime}$ is the space of all continuous linear functionals on $S$. An important relation to remember is $D \subset S \subset S^{\prime} \subset D^{\prime}$. All the GFs in the space $S^{\prime}$ have generalized Fourier transform given by the relation $\hat{F}[\phi]=F[\hat{\phi}]$ where now $\phi \epsilon S$. We will say no more on this interesting subject here. See the references at the end of this paper. We strongly recommend the book by Strichartz ${ }^{10}$ for this subject.

2- Differentiation of GFs- This is the most important operation on GFs because of its many applications in mathematics. Many of the problems of wave propagation need the use of this concept. Let us start with a $C^{1}$ function $f(x)$ which we identify with the functional $F[\phi]$. The functional representation of $f^{\prime}(x)$ can be manipulated as follows 


$$
F^{\prime}[\phi]=\int f^{\prime}(x) \phi(x) d x=-\int f(x) \phi^{\prime}(x) d x=-F\left[\phi^{\prime}\right]
$$

Note that we have integrated by parts after the second equality sign and we have used the fact that the support of $\phi$ is compact to drop the terms involving the limits of the integral. Since we know that the function $\phi^{\prime}$ is in $D, F\left[\phi^{\prime}\right]$ is a proper functional on $D$ and we can use the following relation for the definition of the generalized derivative (GD) of a GF

$$
F^{\prime}[\phi]=-F\left[\phi^{\prime}\right]
$$

From this we can define the nth generalized derivative of a GF

$$
F^{(n)}[\phi]=(-1)^{n} F\left[\phi^{(n)}\right]
$$

This significant result states that GFs have GDs of all orders. When we work with symbolic functions we often utilize a bar over a derivative operator to designate generalized differentiation if the operation can be confused with ordinary differentiation. See eqs. (30) and (34) below. It is understood that the differentiation of singular GFs can only be GD and in that case we will not use this notation.

Example 10- GD of the Heaviside function $h(x)=0$ for $x<0$ and $h(x)=1$ for $x>0$ is found as follows

$$
H^{\prime}[\phi]=-H\left[\phi^{\prime}\right]=-\int_{0}^{\infty} \phi^{\prime}(x) d x=\phi(0)
$$

Note that we have used the fact that the test function has a bounded support and, thus, we have $\phi(\infty)=0$. Therefore, we have

$$
\bar{h}^{\prime}(x)=\delta(x)
$$

That is, the GD of the Heaviside function is the Dirac delta function. Note that $h^{\prime}(x)=0$ but $\bar{h}^{\prime}(x) \neq 0$. We will return to this point later. This result shows that the GD of an OF can be a singular GF!

Example 11- Generalized derivative of the Dirac delta function is

$$
\delta^{\prime}[\phi]=\int \delta^{\prime}(x) \phi(x) d x=-\delta\left[\phi^{\prime}\right]=-\phi^{\prime}(0)
$$

Similarly, we have

$$
\delta^{(n)}[\phi]=\int \delta^{(n)}(x) \phi(x) d x=(-1)^{n} \phi^{(n)}(0)
$$

Note that in eqs. (31) and (32), the integrals are meaningless and stand for the functional rules to the left of the first equality sign in the respective equations.

Example 12- Let the OF $f(x)$ be differentiable with a single jump of $\Delta f=f\left(x_{0+}\right)-f\left(x_{0_{-}}\right)$at the point $x_{0}$ as shown in Figure 5. 


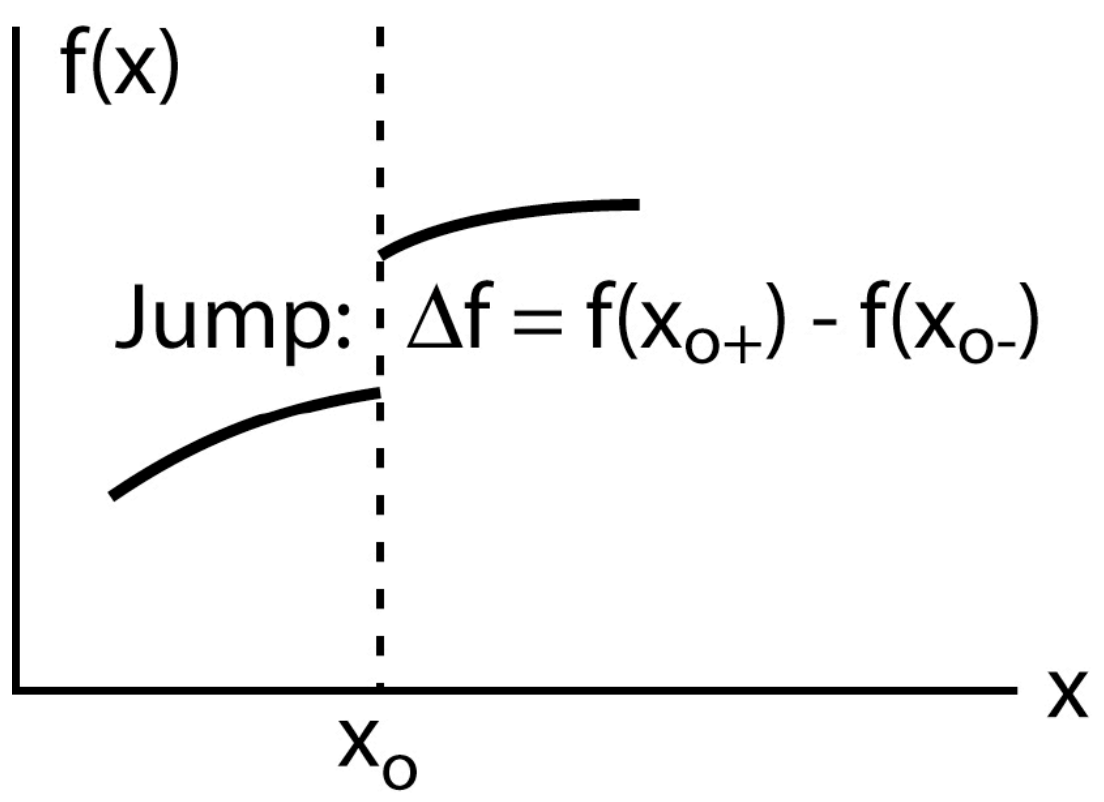

Figure 5- A differentiable function with a single jump discontinuity at $x_{0}$

We find the GD of this function as follows. Let $F[\phi]$ be the functional representation of this function. Then its GD can be manipulated as follows

$$
\begin{aligned}
F^{\prime}[\phi]= & -F\left[\phi^{\prime}\right]=-\int f(x) \phi^{\prime}(x) d x=-\int_{-\infty}^{x_{0-}} f(x) \phi^{\prime}(x) d x-\int_{x_{0+}}^{\infty} f(x) \phi^{\prime}(x) d x= \\
& \int_{-\infty}^{x_{0-}} f^{\prime}(x) \phi(x) d x+\int_{x_{0+}}^{\infty} f^{\prime}(x) \phi(x) d x+\left(f\left(x_{0+}\right)-f\left(x_{0-}\right)\right) \phi\left(x_{0}\right)= \\
& \int_{-\infty}^{\infty} f^{\prime}(x) \phi(x) d x+\Delta f \phi\left(x_{0}\right)=\int_{-\infty}^{\infty}\left(f^{\prime}(x)+\Delta f \delta\left(x-x_{0}\right)\right) \phi(x) d x
\end{aligned}
$$

Interpreted in symbolic function notation, we have obtained the following important result

$$
\bar{f}^{\prime}(x)=f^{\prime}(x)+\Delta f \delta\left(x-x_{0}\right)
$$

Again we have shown that the GD of an $O F$ can be a singular GF. Furthermore, this result shows the need for a notation for generalized differentiation which we have utilized here, i.e., the overbar on the left side.

Example 13- Referring to Figure 4, let the function $k(x)$ be a differentiable function with a jump discontinuity across the surface $f=0$. We define the jump of the function $k(x)$ across the surface as follows:

$$
\triangle k\left(u^{1}, u^{2}\right)=k\left(u^{1}, u^{2}, 0_{+}\right)-k\left(u^{1}, u^{2}, 0_{-}\right) \equiv k\left(\left.\boldsymbol{x}\right|_{f=0_{+}}\right)-k\left(\left.\boldsymbol{x}\right|_{f=0_{-}}\right)
$$

where $\boldsymbol{u}=\left(u^{1}, u^{2}, u^{3}\right)$ is the curvilinear coordinate system we used in Example 7. Note that the function $k(x)$ is discontinuous in variable $u^{3}=f$ only. This is precisely why we selected such a coordinate 
system in the vicinity of the surface of discontinuity. Now we are going to use eq. (34) to derive the following important result:

$$
\begin{aligned}
& \frac{\bar{\partial} k(\boldsymbol{x}(\boldsymbol{u}))}{\partial x_{i}}= \\
& \frac{\partial u^{j}}{\partial x_{i}} \frac{\partial k}{\partial u^{j}}+\Delta k \frac{\partial u^{3}}{\partial x_{i}} \delta\left(u^{3}\right)=\frac{\partial k}{\partial x_{i}}+\Delta k \frac{\partial f}{\partial x_{i}} \delta(f)=\frac{\partial k}{\partial x_{i}}+\Delta k n_{i} \delta(f)
\end{aligned}
$$

We have used the summation convention on repeated index in the above equation. We can write the above result in vector notation as follows:

$$
\bar{\nabla} k(\boldsymbol{x})=\nabla k(x)+\Delta k \boldsymbol{n} \delta(f) \quad \text { (Note } \boldsymbol{n}=\nabla f \text { here.) }
$$

We give here two more important relations which can be remembered easily. Let $\boldsymbol{k}(\boldsymbol{x})$ be a vector field with a jump discontinuity across the surface $f=0$ with the jump defined similar to eq. (35). Then the following results hold:

$$
\begin{aligned}
& \bar{\nabla} \cdot \boldsymbol{k}(\boldsymbol{x})=\nabla \cdot \boldsymbol{k}(\boldsymbol{x})+\Delta \boldsymbol{k} \cdot \boldsymbol{n} \delta(f) \quad \text { (Note } \boldsymbol{n}=\nabla f \text { here.) } \\
& \bar{\nabla} \times \boldsymbol{k}(\boldsymbol{x})=\nabla \times \boldsymbol{k}(\boldsymbol{x})+\boldsymbol{n} \times \Delta \boldsymbol{k} \delta(f) \quad \text { (Note } \boldsymbol{n}=\nabla f \text { here.) }
\end{aligned}
$$

With what we have obtained so far we can solve a lot of interesting problems. Before we give some examples, let us say a little more about why one should use generalized derivative.

\section{4- Why We Use Generalized Derivatives}

The most important fact to remember about GDs is that in GF theory generalized differentiation is a continuous operation. In particular, generalized differentiation and integration are inverse operations. This means that the integration of GD of a GF recovers the function faithfully with all of its jumps. Examine the following

Example 14- Let $h(x)$ be the Heaviside function defined in Example 10. Then we can easily show that

$$
\int_{-\infty}^{x} \bar{h}^{\prime}(y) d y=h(x) \text { but } \int_{-\infty}^{x} h^{\prime}(y) d y=\text { constant } \neq h(x)
$$

Therefore, any problem involving unknowns with discontinuities should be set up in GF space and all derivatives must be treated as GDs from the start. Such discontinuities are either artificially introduced, e.g., as in FW-H equation, or are genuine, e.g., a shock wave in the flow field. The statement following eq. (40) has profound implications for applications some of which will mentioned below:

1- All local conservation laws obtained from control volume analysis are valid if all the derivatives are considered GDs. Examples of such conservation laws are the mass continuity and the NavierStokes equations.

2- The jump conditions across a natural discontinuity in the flow are inherent in the conservation laws. These jump conditions are obtained easily by applying the definition of GD to the conservation laws. See Example 15 below. 
3- Any problem involving derivatives in which singular GFs appear must be set up in GF space. One must then carefully distinguish between the GDs and the ordinary derivatives of any function appearing in the algebra. An example of such a problem is finding the Green's function of an ODE or a PDE.

4- Generalized differentiation operator commutes with all common limit operations. This is the most pleasant and useful property of generalized differentiation. The following operations are allowed provided that the limits of the integration is independent of the variable of differentiation:

$$
\frac{\bar{\partial}}{\partial x_{i}} \int_{\Omega} q(\boldsymbol{x}, \boldsymbol{y}) d \boldsymbol{y}=\int_{\Omega} \frac{\bar{\partial} q(\boldsymbol{x}, \boldsymbol{y})}{\partial x_{i}} d \boldsymbol{y}, \quad \frac{\bar{\partial}}{\partial x_{i}} \lim _{n \rightarrow \infty} q_{n}(\boldsymbol{x}, \boldsymbol{y})=\lim _{n \rightarrow \infty} \frac{\bar{\partial} q_{n}(\boldsymbol{x}, \boldsymbol{y})}{\partial x_{i}}
$$

See the NASA paper by Farassat ${ }^{31}$ for more examples.

Example 15- Let us find one of the jump conditions across an unsteady shock wave which is described by the surface $f(\boldsymbol{x}, t)=0$. We define the jumps in fluid parameters as in eq. (35). The mass continuity equation is

$$
\frac{\partial \rho}{\partial t}+\nabla \cdot(\rho \boldsymbol{u})=0
$$

Here the fluid density is $\rho$ and the fluid velocity is $\boldsymbol{u}$. First we know this conservation law was derived from a control volume analysis. Therefore, it is valid if we treat all ordinary derivatives as GDs, i.e., we have

$$
\frac{\bar{\partial} \rho}{\partial t}+\bar{\nabla} \cdot(\rho \boldsymbol{u})=0
$$

We now use the definition of GD in this equation as follows

$$
\begin{aligned}
& \frac{\bar{\partial} \rho}{\partial t}+\bar{\nabla} \cdot(\rho \boldsymbol{u})= \\
& \frac{\partial \rho}{\partial t}+\Delta \rho \frac{\partial f}{\partial t} \delta(f)+\nabla \cdot(\rho \boldsymbol{u})+\Delta(\rho \boldsymbol{u}) \cdot \boldsymbol{n} \delta(f)=\Delta\left[\rho\left(u_{n}-v_{n}\right)\right] \delta(f)=0
\end{aligned}
$$

where we have used the notations $u_{n}=\boldsymbol{u} \cdot \boldsymbol{n}$ and $v_{n}=-\partial f / \partial t$ is the local normal velocity of the shock. We have used eq. (42) in the above result. Therefore, one jump condition across an unsteady shock wave is

$$
\triangle\left[\rho\left(u_{n}-v_{n}\right)\right]=0 \quad \text { or } \quad \triangle\left(\rho u_{n}\right)-v_{n} \Delta \rho=0
$$

Note that we derived this condition without using the pill box analysis employed in the classical derivation of the result.

5- We can use the Green's function technique to find the discontinuous solutions of an ODE or a PDE provided that we formulate the problem in GF space and use generalized differentiation everywhere. This is a very powerful result and its impact on applications is enormous. We will not give any example here but you can see some good examples in the next section.

We conclude this section by stating the fundamental theorem that characterizes GFs in $D^{\prime}$ : 
6- GFs in $D^{\prime}$ are GDs of finite orders of continuous functions ${ }^{7}$. This is a very profound result obtained by Laurent Schwartz. As a simple example we note that the Dirac delta function is the generalized second derivative of the continuous function that is zero on the negative axis and is equal to $x$ on the positive axis.

\section{5- A Note on Test Function Spaces}

We have developed above the GF theory using the test function space $D$. Although the GF spaces $D^{\prime}$ and $S^{\prime}$ are very useful in applications, we often have to use test functions other than the spaces $D$ and $S$ which are not $C^{\infty}$ functions. But the construction of GFs in the space $D^{\prime}$ can be used as a model to get other kinds of GFs. For example if we take a space of test functions consisting of $C^{n}$ functions, then we can define GFs on this space as continuous linear functionals which only have GDs up to the order $n$. We must, of course, modify our definition of the continuity of a linear functional appropriately. To define GFs suitable to treat ODEs and PDEs, say a differential operator L (a differential equation plus linear homogeneous boundary conditions (BCs)), the test functions must have continuous derivatives of some order (related to the order of highest derivative of L) and should satisfy the adjoint BCs. Everything we have learned about GFs here, particularly the operations on GFs, apply as they are. For this reason we do not specifically identify the test function space unless such identification will shed some light on the problem at hand.

\section{3- Some Applications of Generalized Differentiation}

We will give two important applications of generalized differentiation which are very useful in solving wave propagation problems. These are:

1- The Green's function of a second order ODE, and

2- The imbedding of a problem in another problem whose Green's function is known.

You should note that in these applications we have to utilize almost everything we presented on GFs in the previous section.

\section{1- The Green's Function of a Second Order ODE}

In working with wave propagation problems in time or frequency domains, we often have to obtain the Green's function of a second order ODE in intermediate steps. Since we are now able to explain some of the subtleties of the derivation of this Green's function from the point of GF theory, we will present a discussion of it here. We hope that you will agree with us that GF theory is the right tool to use here.

Consider the following second order ODE with two linear homogeneous boundary conditions (BCs):

$$
\begin{aligned}
& l u=a(x) u^{\prime \prime}(x)+b(x) u^{\prime}(x)+c(x) u(x)=k(x) \quad x \in[0,1] \\
& \mathrm{BC}_{1}[u]=0, \quad \mathrm{BC}_{2}[u]=0
\end{aligned}
$$

The Green's function $g(x, y)$ for this problem is formulated classically as follows ${ }^{8}$ (see volume 1 of this reference): 


$$
\begin{aligned}
& l_{x} g(x, y) \equiv a(x) \frac{\partial^{2} g(x, y)}{\partial x^{2}}+b(x) \frac{\partial g(x, y)}{\partial x}+c(x) g(x, y)=\delta(x-y), x, y \in[0,1] \\
& \mathrm{BC}_{1}[g(x, y)]=0, \quad \mathrm{BC}_{2}[g(x, y)]=0 \quad \text { (in variable } x \text { only) }
\end{aligned}
$$

Now we see that we have the Dirac delta function on the right of eq. (48) which is a singular GF. So the partial derivatives in this equation are most likely GDs (we know the answer but we are presenting what most books for engineers and physicists give). But how did we suddenly get into the GF space? Well, we did not really set up our problem correctly. First from the theory of ODEs we must know something about the nature of the solution of the BC problem described by eqs.(46) and (47). Let us assume that we know that $u \in C^{2}$. This can be proved a posteriori when the solution is at hand. In that case, in the domain of the definition of this function, since a $C^{2}$ function cannot have any jumps or sharp corners, we have

$$
\bar{l} u \equiv a(x) \bar{u}^{\prime \prime}(x)+b(x) \bar{u}^{\prime}(x)+c(x) u(x)=a(x) u^{\prime \prime}(x)+b(x) u^{\prime}(x)+c(x) u(x)=l u
$$

The Green's function is used to find the unknown function from the following relation:

$$
u(x)=\int_{0}^{1} k(y) g(x, y) d y
$$

Let us discover further properties of the Green's function from this relation by applying the linear differential operator (the ODE plus the BCs) to both sides of the equality. First we get

$$
l u(x)=\bar{l} u(x)=\bar{l}_{x} \int_{0}^{1} k(y) g(x, y) d y=\int_{0}^{1} k(y) \bar{l}_{x} g(x, y) d y=k(x)
$$

Note that we freely took the generalized derivatives in $\bar{l}_{x}$ inside the integral sign without concern because this step is allowed in GF theory. From this we immediately can see that

$$
\bar{l}_{x} g(x, y)=a(x) \frac{\bar{\partial}^{2} g(x, y)}{\partial x^{2}}+b(x) \frac{\bar{\partial} g(x, y)}{\partial x}+c(x) g(x, y)=\delta(x-y)
$$

Therefore, we established rigorously that all the derivatives in eq. (48) are GDs. We will get back to the implications of this fact soon. Next we apply the two BCs to both sides of eq. (51) remembering that these are linear and homogeneous:

$$
\mathrm{BC}_{x}[u(x)]=\mathrm{BC}_{x} \int_{0}^{1} k(y) g(x, y) d y=\int_{0}^{1} k(y) \mathrm{BC}_{x}[g(x, y)] d y=0
$$

where the symbol $\mathrm{BC}_{x}$ stands for either of the two BCs but applying to variable $x$ only. From the above result, because the function $k(y)$ is arbitrary, we conclude that the Green's function in variable $x$ satisfies both BCs:

$$
\mathrm{BC}_{1, x}[g(x, y)] \text { and } \mathrm{BC}_{2, x}[g(x, y)]
$$


Now let us see what eq. (53) implies about the Green's function. Obviously this function changes its character at $x=y$. So let us assume that it is described as follows by two $C^{2}$ functions with possible jumps in their values and their derivatives at $x=y$ :

$$
g(x, y)= \begin{cases}g_{1}(x, y) & x<y \\ g_{2}(x, y) & x>y\end{cases}
$$

First of all since the Dirac delta function $\delta(x-y)$ is identically zero in the two open intervals on both sides of $x=y$, we must have:

$$
l_{x} g_{1}(x, y)=l_{x} g_{2}(x, y)=0
$$

This means that the functions $g_{1}(x, y)$ and $g_{2}(x, y)$ in variable $\mathrm{x}$ are solutions of the homogeneous ODE $l_{x} g(x, y)=0$. Let us take the GD of the first and second order of the Green's function described by eq. (56)

$$
\begin{gathered}
\frac{\bar{\partial} g(x, y)}{\partial x}=\frac{\partial g(x, y)}{\partial x}+\left[g_{2}\left(y_{+}, y\right)-g_{1}\left(y_{-}, y\right)\right] \delta(x-y) \\
\frac{\bar{\partial}^{2} g(x, y)}{\partial x^{2}}=\frac{\partial^{2} g(x, y)}{\partial x^{2}}+\left[\frac{\partial g_{2}\left(y_{+}, y\right)}{\partial x}-\frac{\partial g_{1}\left(y_{-}, y\right)}{\partial x}\right] \\
\delta(x-y)+\left[g_{2}\left(y_{+}, y\right)-g_{1}\left(y_{-}, y\right)\right] \delta^{\prime}(x-y)
\end{gathered}
$$

We must substitute these two relations in eq. (53) and equate both sides term by term. Since there is no derivative of the Dirac delta function on the right of eq. (53), we immediately conclude that

$$
g_{2}\left(y_{+}, y\right)-g_{1}\left(y_{-}, y\right)=0
$$

This means that the Green's function is continuous at $x=y$. Furthermore, we get

$$
\left[\frac{\partial g_{2}\left(y_{+}, y\right)}{\partial x}-\frac{\partial g_{1}\left(y_{-}, y\right)}{\partial x}\right]=\frac{1}{a(y)} \quad(\text { assuming that } a(y) \neq 0 \text { for } y \in[0,1])
$$

This means that the derivative of the Green's function with respect to $x$ has a jump described by the above equation at $x=y$. We can show that eqs. (55), (57), (60) and (61) uniquely define the Green's function. Note that only by using GF theory we have been able to get the above results rigorously and satisfactorily.

Example 16- Let us take $l u(x)=u^{\prime \prime}$ for $x \in[0,1]$ with the following BCs:

$$
u(0)-2 u^{\prime}(0)=0 \text { and } u(1)+u^{\prime}(1)=0
$$

The above procedure will give the following Green's function for this problem:

$$
g(x, y)= \begin{cases}\frac{(y-2)(x+2)}{4} & x<y \\ \frac{(y+2)(x-2)}{4} & x>y\end{cases}
$$


This Green's function satisfies the conditions derived above. Note that the differential operator in this example is selfadjoint and, therefore, the symmetry of the Green's function in variables $x$ and $y$ is expected ${ }^{8}$.

\section{2- The Imbedding of a Problem in Another Problem}

We got interested in this problem through our work on the Ffowcs Williams-Hawkings (FW-H) equation $^{2}$. The situation is described in Figure 6 below.

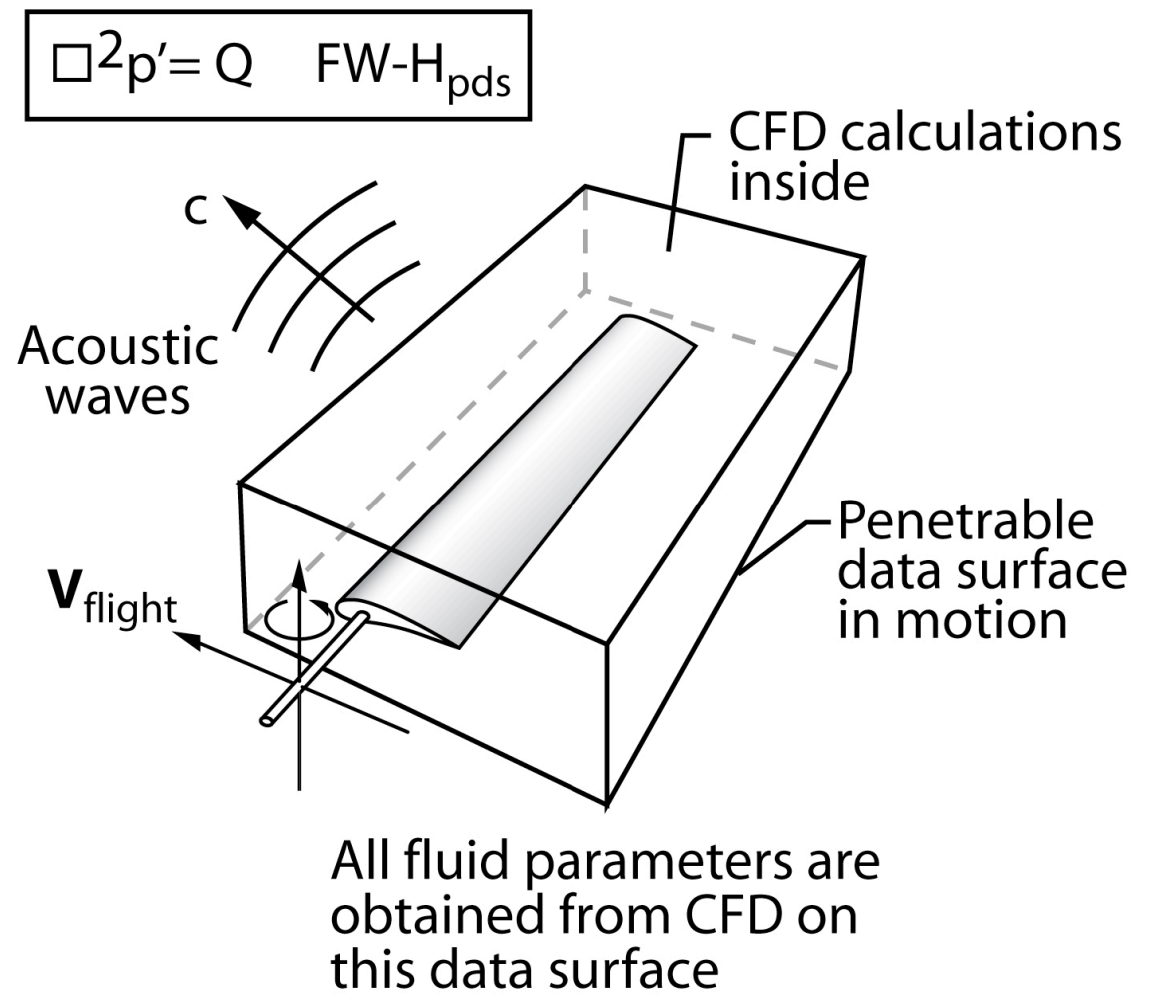

Figure 6- The problem of noise radiation from a moving body (a helicopter rotor blade here) as formulated by Ffowcs Williams and Hawkings

We are given an inhomogeneous wave equation in the exterior of a moving surface described implicitly by the function $f(\boldsymbol{x}, t)=0$. This surface will be called the data surface here. Ffowcs Williams and Hawkings searched for a radiation formula in the exterior of the data surface. Essentially, these authors used an imbedding method by making their wave equation valid in the entire unbounded space ${ }^{2}$. This imbedding allowed the use of the free space Green's function for getting the radiation formula. They assumed that the fluid inside the data surface is always at rest with the condition of the undisturbed medium. Since an artificial discontinuity has been introduced in the medium, one must work with GFs. This imbedding technique provides us with an elegant method of deriving a radiation formula. This technique can be applied to a whole range of other problems involving ODEs and PDEs particularly those related to wave propagation. We will discuss some of these problems here. First, however, we present the imbedding technique. 


\subsection{1- The Imbedding Technique}

We will consider the problem of radiation from a moving and deformable surface $f(\boldsymbol{x}, t)=0$, assuming that $\nabla f=\boldsymbol{n}$, where $\boldsymbol{n}$ is the unit outward normal. We have shown the moving surface at two times in space in Figure 7.
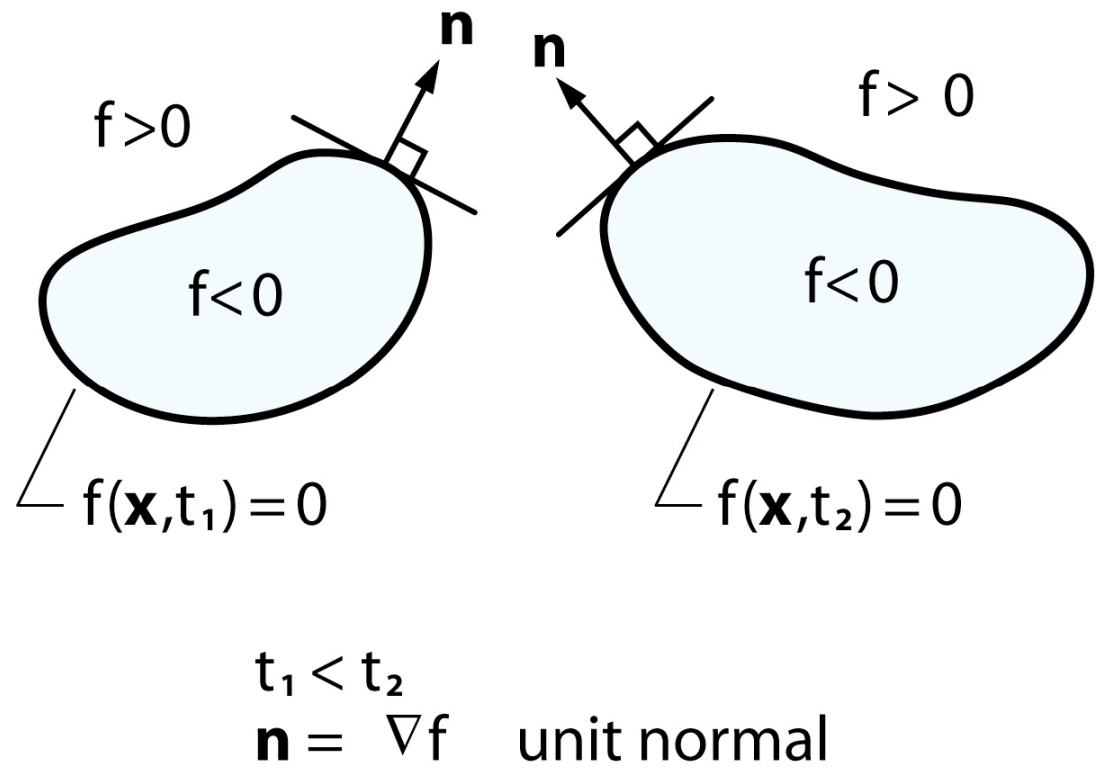

Figure 7- A moving deformable surface described by $f(x, t)=0$ at two different times $t_{1}$ and $t_{2}$ in space

We want to solve the following exterior acoustic radiation problem:

$$
\begin{aligned}
& \frac{1}{c^{2}} \frac{\partial^{2} \varphi}{\partial t^{2}}-\nabla^{2} \varphi= \\
& \square^{2} \varphi=Q(x, t) \quad(x \text { in exterior of } f=0, c=\text { constant })
\end{aligned}
$$

We are assuming that we have all the data about the function $\varphi$ on the moving surface. These data may be overdetermined but right now we are not concerned about this fact. We want to extend the domain of the problem to the interior of the surface so that we can use the simple free space Green's function of the wave equation. In other words, we want to imbed our problem in another problem with larger domain in space (this can be done also for the dimension of time). This imbedding will require the powerful machinery of GFs. Here is how we do the imbedding. We start by extending the definition of the two functions in eq. (64) as follows:

$$
\tilde{\varphi}(\boldsymbol{x}, t)=\left\{\begin{array}{ll}
\varphi(\boldsymbol{x}, t) & f>0 \\
0 & f<0
\end{array}, \text { and } \tilde{Q}(\boldsymbol{x}, t)= \begin{cases}Q(\boldsymbol{x}, t) & f>0 \\
0 & f<0\end{cases}\right.
$$

The first thing we notice is that with this extension, the new functions satisfy the following wave equation:

$$
\square^{2} \tilde{\varphi}=\tilde{Q}(\boldsymbol{x}, t)
$$


Note that the unknown function $\tilde{\varphi}$ is discontinuous. To find this function, we must set the problem in GF space. This simply means that we find what the wave operator looks like when all the ordinary derivatives are written as GDs as follows:

$$
\frac{\bar{\partial} \tilde{\varphi}}{\partial t}=\frac{\partial \tilde{\varphi}}{\partial t}+\varphi \frac{\partial f}{\partial t} \delta(f)=\frac{\partial \tilde{\varphi}}{\partial t}-\varphi v_{n} \delta(f)
$$

where $v_{n}=-\partial f / \partial t$ is local normal velocity of the surface which is unambiguously defined even for a deformable surface. Also we use the notation $\varphi=\varphi\left(f=0_{+}\right)$when this function multiplies the Dirac delta function $\delta(f)$ as in the last term on the right of eq. (67). Next we find the following relations:

$$
\begin{aligned}
& \frac{\bar{\partial}^{2} \tilde{\varphi}}{\partial t^{2}}=\frac{\partial^{2} \tilde{\varphi}}{\partial t^{2}}-\frac{\partial \varphi}{\partial t} v_{n} \delta(f)-\frac{\partial}{\partial t}\left[\varphi v_{n} \delta(f)\right] \\
& \nabla^{2} \tilde{\varphi}=\nabla^{2} \tilde{\varphi}+\boldsymbol{n} \cdot \nabla \varphi \delta(f)+\nabla \cdot[\varphi \boldsymbol{n} \delta(f)]
\end{aligned}
$$

where we have again used the notation that any function of $\tilde{\varphi}$ multiplying the Dirac delta function is evaluated at $f=0_{+}$. Let us define the local normal Mach number of the surface as $M_{n}=v_{n} / c$, and define additionally the following two symbols $\varphi_{t}=\partial \varphi / \partial t$ and $\varphi_{n}=\boldsymbol{n} \cdot \nabla \varphi$. Then the above two relation will give us:

$$
\bar{\square}^{2} \tilde{\varphi}=\square^{2} \tilde{\varphi}-\frac{1}{c} \varphi_{t} M_{n} \delta(f)-\frac{1}{c} \frac{\partial}{\partial t}\left[\varphi M_{n} \delta(f)\right]-\varphi_{n} \delta(f)-\nabla \cdot[\varphi \boldsymbol{n} \delta(f)]
$$

Or, after using eq. (66), we obtain

$$
\bar{\square}^{2} \tilde{\varphi}=-\frac{1}{c} \varphi_{t} M_{n} \delta(f)-\frac{1}{c} \frac{\partial}{\partial t}\left[\varphi M_{n} \delta(f)\right]-\varphi_{n} \delta(f)-\nabla \cdot[\varphi \boldsymbol{n} \delta(f)]
$$

Note that we do need to use a bar over the wave operator on the left side since from the right side it is clear that all derivatives involved are GDs. Now we can use the Green's function of the wave equation in the unbounded space, the so-called free-space Green's function, to find the unknown function $\tilde{\varphi}(\boldsymbol{x}, t)$ everywhere in space. The result is the Kirchhoff formula for moving surfaces ${ }^{31,32}$. The classical derivation of this formula by Morgans ${ }^{33}$ is very complicated and in fact until Farassat and Myers rederived it by the modern method presented here, there were some doubts expressed about the correctness of Morgans' result ${ }^{2,34}$.

We will not derive the Kirchhoff formula for moving surfaces here since the two references by Farassat and Myers ${ }^{32}$ and Farassat ${ }^{31}$ are quite comprehensive alleviating the need for further elaboration. However, we next derive some classical results for stationary surfaces by the imbedding method which are surprisingly simpler than the classical methods of their derivation.

Before we end this discussion we want to mention an important question that comes to mind. Answering this question may lead to further applications of the Green's function of the unbounded space. To imbed our exterior radiation problem in $\mathbb{R}^{3}$ we have imposed that inside the surface both $\tilde{\varphi}$ and $\tilde{Q}$ be zero. Can the functions $\tilde{\varphi}$ and $\tilde{Q}$ be assumed to take other values and are there advantages in doing so? We will find the answer to this question using an ODE to simplify the analysis. 


\subsection{2- Derivation of Some Classical Results Using the Imbedding Technique}

1- The Green's Theorem for Laplace Equation in the Exterior Domain of a Surface- The problem is described in Figure 8.

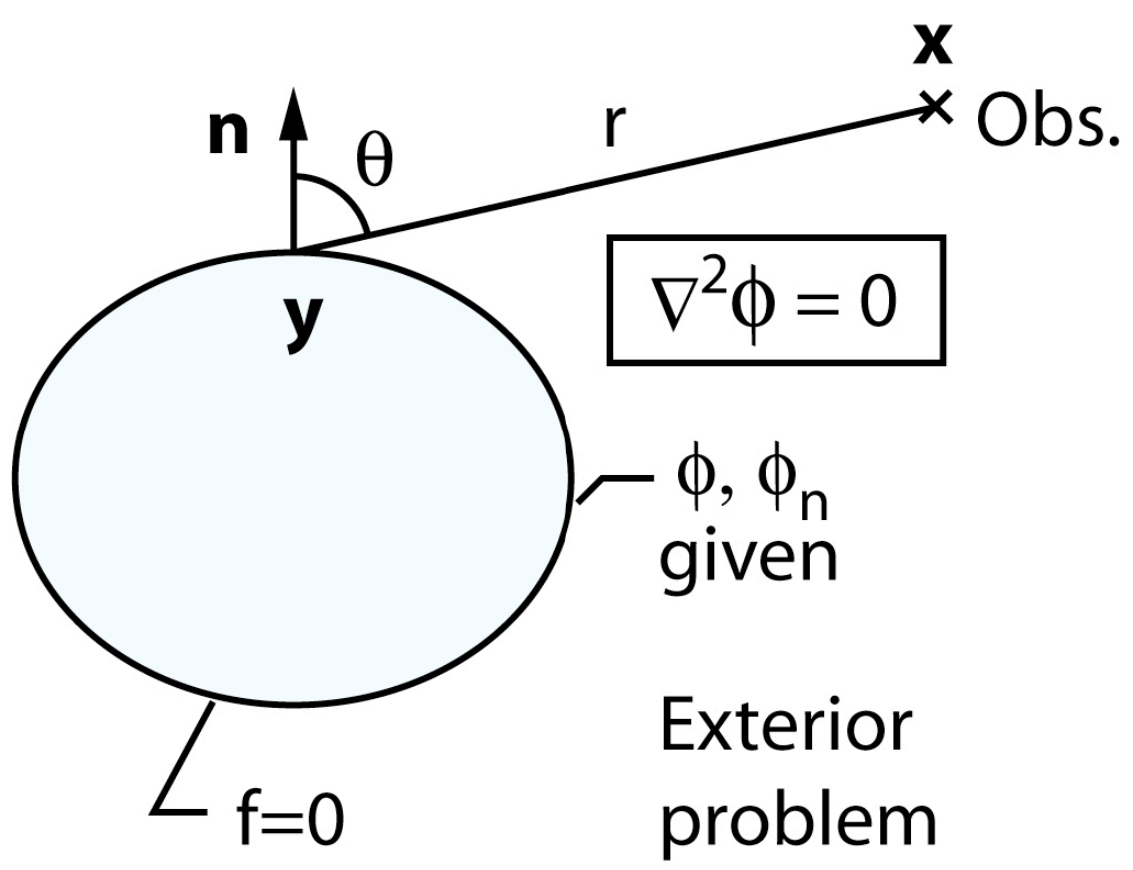

Figure 8- Problem description for deriving the Green's theorem for Laplace equation, $f>0$ outside the surface and $n=\nabla f$ on the surface

We imbed this problem in $\mathbb{R}^{3}$ by extending the definition of $\varphi$ to the interior of the surface as in eq. (65). We find generalized Laplace equation next:

$$
\bar{\nabla}^{2} \tilde{\varphi}=\nabla^{2} \tilde{\varphi}+\boldsymbol{n} \cdot \nabla \varphi \delta(f)+\nabla \cdot[\varphi \boldsymbol{n} \delta(f)]=\varphi_{n} \delta(f)+\nabla \cdot[\varphi \boldsymbol{n} \delta(f)] \quad\left(x \in \mathbb{R}^{3}\right)
$$

The definition of all symbols are identical to those in Section 3.2.1. We have used the fact that $\nabla^{2} \tilde{\varphi}=0$ in $\mathbb{R}^{3}$. The Green's function of this problem in the unbounded space is $-1 /(4 \pi r)$, where $r=|\boldsymbol{x}-\boldsymbol{y}|$. Here $\boldsymbol{x}$ and $\boldsymbol{y}$ are the observer and the source (field) variables, respectively. The solution of the above equation will give us the answer we are seeking as follows:

$$
\begin{gathered}
4 \pi \tilde{\varphi}(\boldsymbol{x})=-\int_{\mathbb{R}^{3}} \frac{\varphi_{n}(\boldsymbol{y})}{r} \delta(f) d \boldsymbol{y}-\nabla \cdot \int_{\mathbb{R}^{3}} \frac{\varphi(\boldsymbol{y}) \boldsymbol{n}}{r} \delta(f) d \boldsymbol{y}= \\
-\int_{\mathbb{R}^{3}} \frac{\varphi_{n}(\boldsymbol{y})}{r} \delta(f) d \boldsymbol{y}-\int_{\mathbb{R}^{3}} \varphi(\boldsymbol{y}) \boldsymbol{n} \cdot \nabla_{x}\left(\frac{1}{r}\right) \delta(f) d \boldsymbol{y}= \\
\int_{f=0}\left(-\frac{\varphi_{n}(\boldsymbol{y})}{r}+\frac{\varphi \cos \theta}{r^{2}}\right) d S
\end{gathered}
$$

We have used the result of Example 7, i.e., eq. (13), twice in the last step. The angle $\theta$ is between the local outward normal to the surface and $\boldsymbol{r}=\boldsymbol{x}-\boldsymbol{y}$, see Figure 8. This is the well-known Green's theo- 
rem for the Laplace equation. Note that a bonus of the imbedding method is that we know that by construction this equation will give $\tilde{\varphi}=0$ inside the surface, a fact not at all obvious in classical derivation and must be established separately.

2- The Classical Kirchhoff Formula (The Exterior Problem)- We will now give the derivation of this well-known result based on eq. (71), i.e. using the imbedding method. Referring to Figure 9, we imbed the problem in $\mathbb{R}^{3}$ and use the fact that $M_{n}=0$ in eq. (71) to get:

$$
\bar{\square}^{2} \tilde{\varphi}=-\varphi_{n} \delta(f)-\nabla \cdot[\varphi \boldsymbol{n} \delta(f)]
$$

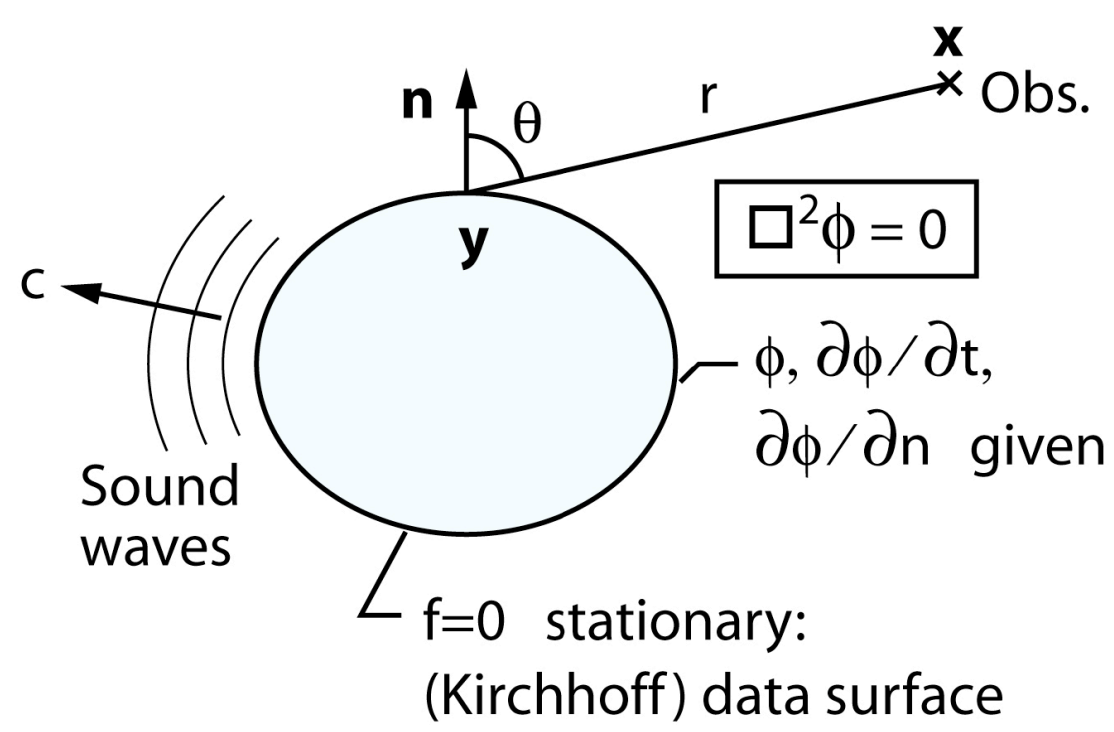

Figure 9- Problem description for deriving the classical Kirchhoff formula, $f>0$ outside the surface and $n=\nabla f$ on the surface

The Green's function of the wave equation in the unbounded three dimensional space is:

$$
G(\boldsymbol{x}, t ; \boldsymbol{y}, \tau)= \begin{cases}0 & \tau>t \\ \delta(\tau-t+r / c) / 4 \pi r & \tau \leqslant t\end{cases}
$$

where $r=|\boldsymbol{x}-\boldsymbol{y}|$. Here $(\boldsymbol{x}, t)$ and $(\boldsymbol{y}, \tau)$ are the observer and the source space-time variables, respectively. We usually use the following symbol: $g=\tau-t+r / c$.

We are first interested in the solution of the equation:

$$
\bar{\square}^{2} \psi=q(x, t) \delta(f)
$$

The solution of eq. (76) is $8,9,29$ :

$$
4 \pi \psi(\boldsymbol{x}, t)=\int q(\boldsymbol{y}, \tau) \delta(f) \frac{\delta(g)}{r} d \boldsymbol{y} d \tau=\int_{f=0} \frac{q(\boldsymbol{y}, t-r / c)}{r} d S
$$

The limits of the integral in this equation are given below: 


$$
\int \ldots . . d \boldsymbol{y} d \tau=\int_{-\infty}^{t} \int_{\mathbb{R}^{3}} \ldots . . d \boldsymbol{y} d \tau=\int_{-\infty}^{t} \int_{-\infty}^{\infty} \int_{-\infty}^{\infty} \int_{-\infty}^{\infty} \ldots . . d y_{1} d y_{2} d y_{3} d \tau
$$

Therefore, the solution of eq. (74) is:

$$
\begin{gathered}
4 \pi \tilde{\varphi}(\boldsymbol{x}, t)=-\int_{f=0} \frac{\varphi_{n}(\boldsymbol{y}, t-r / c)}{r} d S-\nabla_{x} \cdot \int_{f=0} \frac{\boldsymbol{n} \varphi(\boldsymbol{y}, t-r / c)}{r} d S= \\
-\int_{f=0} \frac{\varphi_{n}(\boldsymbol{y}, t-r / c)}{r} d S-\int_{f=0} \boldsymbol{n} \cdot \nabla_{x}\left(\frac{\varphi(\boldsymbol{y}, t-r / c)}{r}\right) d S
\end{gathered}
$$

Taking the partial derivatives with respect to the variable $x$ of the integrand of the second integral, we obtain the following result:

$$
\begin{aligned}
& 4 \pi \tilde{\varphi}(\boldsymbol{x}, t)= \\
& \int_{f=0} \frac{c^{-1} \cos \theta \varphi_{t}(\boldsymbol{y}, t-r / c)-\varphi_{n}(\boldsymbol{y}, t-r / c)}{r} d S+\int_{f=0} \frac{\cos \theta \varphi(\boldsymbol{y}, t-r / c)}{r^{2}} d
\end{aligned}
$$

The angle $\theta$ is between the local outward normal to the surface and $\boldsymbol{r}=\boldsymbol{x}-\boldsymbol{y}$, see Figure 9 . This is the well-known classical Kirchhoff formula ${ }^{34-36}$. Note that we know by construction that $\tilde{\varphi}=0$ inside the surface. This fact is not obvious in the classical derivation and, as in the case of the classical derivation of the Green's theorem for the Laplace equation, must be established separately. Notice also that in our derivation we did not have to resort to the four-dimensional Green's theorem for the wave equation. This is a great advantage of the imbedding method over the classical derivation.

3- Radiation into Half Space- The Rayleigh Integral- We will derive two well-known results for radiation into half space by the imbedding method. Let the half space be defined by $x_{3}>0$. We are, therefore, interested in solving the following problem:

$$
\square^{2} \varphi=Q(x, t) \quad\left(x_{3}>0\right)
$$

We will say nothing about the $\mathrm{BC}$ now because two possible $\mathrm{BCs}$ emerge from the imbedding method. Let us imbed this problem in $\mathbb{R}^{3}$ in two ways as follows:

$$
\begin{gathered}
\tilde{\varphi}(\boldsymbol{x}, t)= \begin{cases}\varphi(\boldsymbol{x}, t) & x_{3}>0 \\
-\varphi(\boldsymbol{x}, t) & x_{3}<0\end{cases} \\
\tilde{Q}(\boldsymbol{x}, t)=\left\{\begin{array}{ll}
Q(\boldsymbol{x}, t) & x_{3}>0 \\
-Q(\boldsymbol{x}, t) & x_{3}<0
\end{array} \quad\right. \text { (Imbedding 1) }
\end{gathered}
$$

and

$$
\begin{array}{r}
\tilde{\varphi}(\boldsymbol{x}, t)= \begin{cases}\varphi(\boldsymbol{x}, t) & x_{3}>0 \\
\varphi(\boldsymbol{x}, t) & x_{3}<0\end{cases} \\
\tilde{Q}(\boldsymbol{x}, t)= \begin{cases}Q(\boldsymbol{x}, t) & x_{3}>0 \\
Q(\boldsymbol{x}, t) & x_{3}<0\end{cases}
\end{array}
$$


Imbedding 1- We can show easily that on the plane $x_{3}=0$ the jump $\Delta \tilde{\varphi}=2 \varphi\left(x_{1}, x_{2}, 0_{+}, t\right) \equiv 2 q\left(x_{1}, x_{2}, t\right)$, and also $\Delta\left(\partial \tilde{\varphi} / \partial x_{3}\right)=0$. Therefore, the extended function satisfies the wave equation:

$$
\bar{\square}^{2} \tilde{\varphi}=\tilde{Q}(\boldsymbol{x}, t)-2 q\left(x_{1}, x_{2}, t\right) \delta^{\prime}\left(x_{3}\right) \quad\left(x \in \mathbb{R}^{3}\right)
$$

Using the free space Green's function of the wave equation, we get

$$
\begin{gathered}
4 \pi \tilde{\varphi}(\boldsymbol{x}, t)=\int_{\mathbb{R}^{3}} \frac{\tilde{Q}(\boldsymbol{y}, t-r / c)}{r} d \boldsymbol{y}-\int_{\mathbb{R}^{3}} \frac{2 \delta^{\prime}\left(y_{3}\right) q\left(y_{1}, y_{2}, t-r / c\right)}{r} d \boldsymbol{y}= \\
\int_{\mathbb{R}^{3}} \frac{\tilde{Q}(\boldsymbol{y}, t-r / c)}{r} d \boldsymbol{y}+\int_{\mathbb{R}^{2}} \frac{\partial}{\partial y_{3}}\left[\frac{2 q\left(y_{\left.1, y_{2}, t-r / c\right)}\right]_{y_{3}=0} d y_{1} d y_{2}}{r}\right.
\end{gathered}
$$

When we take the derivative in the integrand of the second integral, we obtain the following result:

$$
\begin{aligned}
& 4 \pi \tilde{\varphi}(\boldsymbol{x}, t)=\int_{\mathbb{R}^{3}} \frac{\tilde{Q}(\boldsymbol{y}, t-r / c)}{r} d \boldsymbol{y}+ \\
& 2 \int_{\mathbb{R}^{2}}\left(\frac{c^{-1} \cos \theta q_{t}\left(y_{1}, y_{2}, t-r / c\right)}{r}+\frac{\cos \theta q\left(y_{1}, y_{2}, t-r / c\right)}{r^{2}}\right) d y_{1} d y_{2}
\end{aligned}
$$

Here we have used $q_{t}=\partial q / \partial t$ and the angle $\theta$ is between the radiation direction $\boldsymbol{x}-\boldsymbol{y}$ and the $x_{3}$ - axis. Note that the function $\tilde{Q}$ is defined in eq. (82). This result is the solution of the following problem in the half space $x_{3}>0$ :

$$
\square^{2} \varphi=Q(\boldsymbol{x}, t), \quad \varphi\left(x_{1}, x_{2}, 0, t\right)=q\left(x_{1}, x_{2}, t\right) \quad\left(x_{3}>0\right)
$$

Imbedding 2- We can show that on the plane $x_{3}=0$, the jump $\Delta \tilde{\varphi}=0$, and also $\Delta\left(\partial \tilde{\varphi} / \partial x_{3}\right)=2 \partial \tilde{\varphi}\left(x_{1}, x_{2}, 0_{+}, t\right) / \partial x_{3} \equiv 2 q\left(x_{1}, x_{2}, t\right)$. Therefore, the extended function satisfies the wave equation:

$$
\bar{\square}^{2} \tilde{\varphi}=\tilde{Q}(\boldsymbol{x}, t)-q\left(x_{1}, x_{2}, t\right) \delta\left(x_{3}\right) \quad\left(x \in \mathbb{R}^{3}\right)
$$

Using the free space Green's function of the wave equation, we get

$$
\begin{aligned}
& 4 \pi \tilde{\varphi}(\boldsymbol{x}, t)=\int_{\mathbb{R}^{3}} \frac{\tilde{Q}(\boldsymbol{y}, t-r / c)}{r} d \boldsymbol{y}-\int_{\mathbb{R}^{3}} \frac{2 \delta\left(y_{3}\right) q\left(y_{1}, y_{2}, t-r / c\right)}{r} d \boldsymbol{y}= \\
& \int_{\mathbb{R}^{3}} \frac{\tilde{Q}(\boldsymbol{y}, t-r / c)}{r} d \boldsymbol{y}-\int_{\mathbb{R}^{2}}\left[\frac{2 q\left(y_{1}, y_{2}, t-r / c\right)}{r}\right]_{y_{3}=0} d y_{1} d y_{2}
\end{aligned}
$$

Since the source in the last integral lies always on the $y_{3}=0$ plane, we can drop this subscript in integrand and we obtain: 


$$
4 \pi \tilde{\varphi}(\boldsymbol{x}, t)=\int_{\mathbb{R}^{3}} \frac{\tilde{Q}(\boldsymbol{y}, t-r / c)}{r} d \boldsymbol{y}-\int_{\mathbb{R}^{2}} \frac{2 q\left(y_{1}, y_{2}, t-r / c\right)}{r} d y_{1} d y_{2}
$$

Note that the function $\tilde{Q}$ is defined in eq. (83).This is the solution of the following problem in the half space $x_{3}>0$ :

$$
\square^{2} \varphi=Q(\boldsymbol{x}, t), \quad \frac{\partial \varphi}{\partial x_{3}}\left(x_{1}, x_{2}, 0, t\right)=q\left(x_{1}, x_{2}, t\right) \quad\left(x_{3}>0\right)
$$

Equation (90) without the volume term is known as the Rayleigh integral ${ }^{36}$.

4- The Imbedding Problem for a Second Order ODE- We consider the second order ODE $l u(x)=u^{\prime \prime}(x)=k(x)$ for $x \in[a, b] \subset[0,1]$ with two linear and homogeneous BCs which we denote as $\mathrm{BC}^{\prime}{ }_{1}[u]=0$ and $\mathrm{BC}^{\prime}{ }_{2}[u]=0$. Assuming that the solution exists, we want to find the solution by imbedding method using the Green's function of the Example 16. The situation is shown in Figure 10.

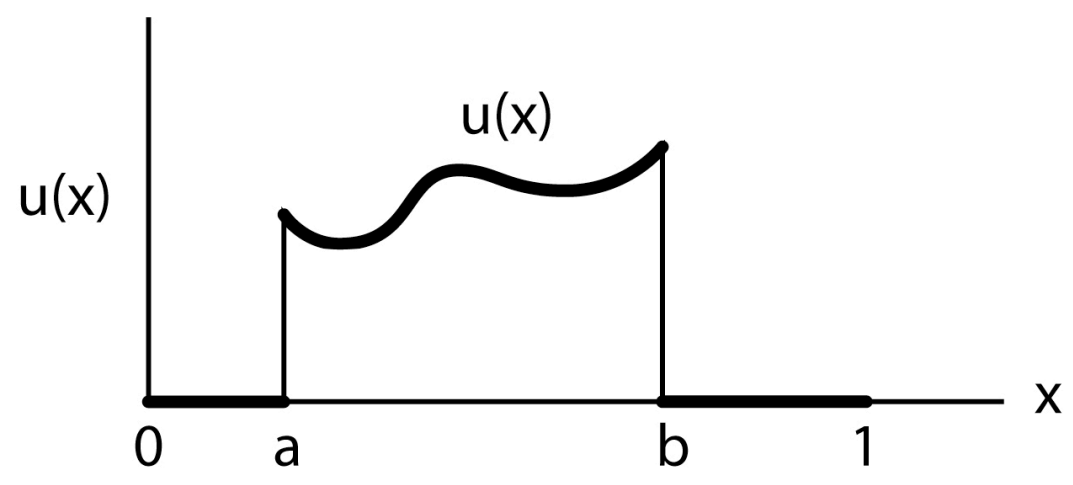

Figure 10- Imbedding an ODE problem for the interval $[a, b]$ in the larger interval $[0,1]$ for the same differential equation

Note that the ODE of Example 16 is the same as that the one on the smaller interval This is required in an imbedding method. Let us extend the unknown function $u(x)$ to the larger interval as follows

$$
\tilde{u}(x)=\left\{\begin{array}{lr}
u(x) & x \in[a, b] \\
0 & x \varepsilon[0,1] \backslash[a, b]
\end{array}, \text { and } \tilde{k}(x)=\left\{\begin{array}{lr}
k(x) & x \in[a, b] \\
0 & x \varepsilon[0,1] \backslash[a, b]
\end{array}\right.\right.
$$

See Figure 10 above. Notice that $\tilde{u}$ satisfies the BCs of Example 16 given by eq. (62). This is required because if this condition is not satisfied, then we cannot use the Green's function of Example 16 given by eq. (63). Note that we have $l \tilde{u}=\tilde{u}^{\prime \prime}=\tilde{f}$. We next find the following result valid on $[0,1]$ :

$$
\begin{aligned}
& \bar{l} \overline{\tilde{u}}(x)= \\
& \overline{\tilde{u}}^{\prime \prime}(x)=\tilde{u}^{\prime \prime}(x)+u^{\prime}(a) \delta(x-a)-u^{\prime}(b) \delta(x-b)+u(a) \delta^{\prime}(x-a)-u(b) \delta^{\prime}(x-b)= \\
& \tilde{k}(x)+u^{\prime}(a) \delta(x-a)-u^{\prime}(b) \delta(x-b)+u(a) \delta^{\prime}(x-a)-u(b) \delta^{\prime}(x-b)
\end{aligned}
$$

Now let $g(x, y)$ be the Green's function of Example 16 given by eq. (63), then we can find a representation of the solution as follows: 


$$
\begin{aligned}
& \tilde{u}(x)= \\
& \quad \int_{0}^{1} \tilde{k}(y) g(x, y) d y+u^{\prime}(a) g(x, a)-u^{\prime}(b) g(x, b)+u(a) \frac{\partial g(x, a)}{\partial y}-u(b) \frac{\partial g(x, b)}{\partial y}= \\
& \quad \int_{a}^{b} k(y) g(x, y) d y+u^{\prime}(a) g(x, a)-u^{\prime}(b) g(x, b)+u(a) \frac{\partial g(x, a)}{\partial y}-u(b) \frac{\partial g(x, b)}{\partial y}
\end{aligned}
$$

We are not finished yet. We can show that using eq. (94), $\tilde{u}\left(a_{-}\right)=0$ and $\tilde{u}\left(a_{+}\right)=u(a)$ give identical relations among the boundary values (BVs) of the imbedded problem. Similarly, we can show that $\tilde{u}\left(b_{+}\right)=0$ and $\tilde{u}\left(b_{-}\right)=u(b)$ also give us another identical relations among the BVs of the imbedded problem. We also have two relations supplied by the BCs of the imbedded problem. These four relations will give us the four unknowns $u(a), u(b), u^{\prime}(a)$, and $u^{\prime}(b)$ which we can then substitute in eq. (94) to completely solve this problem.

Looking at this procedure we note that all we have required of the behavior of the extended function $\tilde{u}(x)$ is that it should satisfy the BCs of the problem whose Green's function is available in the larger domain. This means that any solution $v(x)$ of Example 16 can be assumed in the extended region. Assuming that we have $l v(x)=j(x)$, then eq. (92) must be modified as follows:

$$
\tilde{u}(x)=\left\{\begin{array}{cc}
u(x) & x \in[a, b] \\
v(x) & x \varepsilon[0,1] \backslash[a, b]
\end{array}, \text { and } \quad \tilde{k}(x)=\left\{\begin{array}{cc}
k(x) & x \in[a, b] \\
j(x) & x \varepsilon[0,1] \backslash[a, b]
\end{array}\right.\right.
$$

and eq. (93) becomes

$$
\begin{gathered}
\bar{l} \overline{\tilde{u}}(x)= \\
\overline{\tilde{u}}^{\prime \prime}(x)=\tilde{u}^{\prime \prime}(x)+\left[u^{\prime}(a)-v^{\prime}(a)\right] \delta(x-a)-\left[u^{\prime}(b)-v^{\prime}(b)\right] \delta(x-b)+[u(a)-v(x)] \\
\delta^{\prime}(x-a)-[u(b)-v(b)] \delta^{\prime}(x-b)= \\
\tilde{k}(x)+\left[u^{\prime}(a)-v^{\prime}(a)\right] \delta(x-a)-\left[u^{\prime}(b)-v^{\prime}(b)\right] \delta \\
(x-b)+[u(a)-v(x)] \delta^{\prime}(x-a)-[u(b)-v(b)] \delta^{\prime}(x-b)
\end{gathered}
$$

Again using the Green's function of Example 16, we find the representation of the unknown function as:

$$
\begin{gathered}
\tilde{u}(x)=\int_{0}^{1} \tilde{k}(y) g(x, y) d y+\left[u^{\prime}(a)-v^{\prime}(a)\right] g(x, a)-\left[u^{\prime}(b)-v^{\prime}(b)\right] \\
g(x, b)+[u(a)-v(x)] \frac{\partial g(x, a)}{\partial y}-[u(b)-v(b)] \frac{\partial g(x, b)}{\partial y}
\end{gathered}
$$

Two relations are obtained from $\tilde{u}\left(a_{+}\right)=u(a)$ and $\tilde{u}\left(b_{-}\right)=u(b)$ among the BVs of the imbedded problem. Together with the two BCs of the imbedded problem, we can then find $u(a), u(b), u^{\prime}(a)$, and $u^{\prime}(b)$ and substitute in eq. (97) to find the solution of the imbedded problem.

The implication of this result for wave propagation problems can be substantial. It means that we do not have to assume that fluid inside the moving body is at rest with the conditions of the undisturbed medium in the derivation of the FW-H equation. We can take any solution of the wave equation $\square^{2} \varphi=q$ inside the surface and then apply the imbedding technique. This will allow us to use the free- 
space Green's function and perhaps not have some of the shortcomings of the FW-H equation such as the cancellation between the contributions of the surface and volume integrals.

\section{4- Concluding Remarks}

In this paper we have discussed some applications of GF theory principally to wave propagation problems. We have first given a fairly complete tutorial on GF theory and then have utilized most of the properties of GFs in the examples and applications presented. We have emphasized generalized differentiation here which is a very important operation on GFs with many applications. These are some of our main conclusions:

1- GF theory is a very powerful analytic tool in physics and acoustics with a wide range of applications,

2- The derivation of many classical results in physics and acoustics can be enormously simplified by using GF theory,

3- The power of the Green's function technique is substantially increased if GF theory is utilized as in the imbedding technique, and

4- It is possible to use functions other than the null function inside a moving surface in the imbedding problem used for obtaining the FW-H equation. Some of these choices may alleviate the shortcomings of the FW-H equation and still allow us to use the free-space Green's function.

Generalized function theory is a very powerful tool in mathematics that every physicist and mathematically minded engineer should strive to learn. We have tried to impart to our readers the pleasures of learning and using GFs in their work.

\section{5- References}

1- M. J. Lighthill: On sound generated aerodynamically I. General theory, Proceedings of the Royal Society of London, A211, 1952, 564-587

2- J. E. Ffowcs Williams and D. L. Hawkings: Sound Generated by Turbulence and Surfaces in Arbitrary Motion, Philosophical Transactions of the Royal Society, A264, 1969, 321-342

3- Richard Courant and David Hilbert: Methods of Mathematical Physics, Volume 2, Wiley-Interscience, 1989

4- L. Schwartz: Theorie des Distributions, 2 Volumes, Hermann, Paris, 1957-59

5- L. Hörmander: The Analysis of Linear Partial Differential Operators, 4 volumes, Springer Verlag, 2005-2007

6- Michael E. Taylor: Partial Differential Equations, 3 volumes, Springer Verlag, 1997-2004

7- I. M. Gelfand and G. E. Shilov: Generalized Functions, Volume 1: Properties and Operations, Academic Press, 1964

8- Ivar Stackgold: Boundary Value Problems of Mathematical Physics, 2 volumes, The MacMillan Company, 1968

9- V. S. Vladimirov: Equations of Mathematical Physics, Marcel Dekker, Inc., 1971

10- Robert Strichartz: A Guide to Distribution Theory and Fourier Transforms, CRC Press, 1995 
11- Ram P. Kanwal: Generalized Functions- Theory and Applications, 3rd edition, Birkhauser, 2004

12- D. S. Jones: The Theory of Generalized Functions, 2nd edition, Cambridge University Press, 1982

13- Thomas W. Körner: The Pleasures of Counting, Cambridge University Press, 1996

14- M. J. Lighthill: Introduction to Fourier Analysis and Generalized Functions, Cambridge University Press, 1964

15- Piotr Antosik, Jan Mikusinski and Roman Sikorski: Theory of Distributions- The Sequential Approach, Elsevier Science Publishing Company, 1973

16- Hans Bremermann: Distributions, Complex Variables, and Fourier Transforms, Addison-Wesley Publishing Company, Inc., 1965

17- Jan Mikusinski : Operational Calculus, Volume 1, 2nd edition, Pergamon Press, Inc., 1983

18- Jan Mikusinski and Thomas K. Boehme: Operational Calculus, Volume 2, 2nd edition, Pergamon Press, Inc., 1983

19- S. Fenyo and T. Frey: Modern Mathematical Methods in Technology, Volume 1, American Elsevier Publishing Company, 1969

20- Arthur Erdélyi: Operational Calculus and Generalized Functions, Holt, Reinhart and Winston, Inc., 1962

21- Jean François Colombeau: New Generalized Functions and Multiplication of Distributions, Elsevier Science Publishing Company, 1984

22- Elemér E. Rosinger: Non-Linear Partial Differential Equations- An Algebraic View of Generalized Solutions, Elsevier Science Publishing Company, 1990

23- M. Oberguggenberger: Generalized Solutions of nonlinear Partial Differential Equations, John Wiley and Sons, 1992

24- Robert Goldblatt: Lectures on the Hyperreals: An Introduction to Nonstandard Analysis, Springer Verlag, 1998

25- Roy S. Baty, F. Farassat and John A. Hargreaves:Nonstandard Analysis and Shock Wave Jump Conditions in a One-Dimensional Compressible Gas, Report LA-14334, Los Alamos National Laboratory, May 2007

26- Jesper Lützen: The Prehistory of the Theory of Distributions, Springer-Verlag, 1982

27- Jean Dieudonné: History of Functional Analysis, Elsevier Science Publishing Company, 1981

28- John Sinowiec: Distributions: The Evolution of a Mathematical Theory, Historia Mathematica, 10, $1983,149-183$

29- F. Farassat: Introduction to Generalized Functions With Applications in Aerodynamics and Acoustics, NASA Technical Paper 3428, 1994, (Corrected Copy April 1996), available from NASA Technical Report Server (NTRS) web site, http://ntrs.nasa.gov

30- Alfred Gray, Elsa Abbena and Simon Salamon: Modern Differential Geometry of Curves and Surfaces With MATHEMATICA, Third Edition, Chapman \& Hall/ CRC, 2006 
31- F. Farassat: The Kirchhoff Formulas for Moving Surfaces in Aeroacoustics - The Subsonic and Supersonic Cases, NASA Technical Memorandum 110285, September 1996, available from NASA Technical Report Server (NTRS) web site, http://ntrs.nasa.gov

32- F. Farassat and M. K. Myers: Extension of Kirchhoff's formula to radiation from moving sources, Journal of Sound and Vibration, 123 (3), 1988, 451-460

33- W. R. Morgans: The Kirchhoff formula extended to a moving surface, Philosophical Magazine, 9, 1930, 141-161.

34- D. S. Jones: The Theory of Electromagnetism, Oxford University Press, 1964

35- Philip Morse and Herman Feshbach: Methods of Theoretical Physics, 2 volumes, McGraw-Hill Science, 1953

36- Allan D. Pierce: Acoustics- An Introduction to its Physical Principles and Applications, Acoustical Society of America, 1989 